\title{
SLFN11 captures cancer-immunity interactions associated with platinum sensitivity in ovarian cancer
}

Claudia Winkler ${ }^{1}$, Domenico Ferraioli ${ }^{2}$, Anna Garuti ${ }^{3}$, Federica Grillo ${ }^{4,5}$, Jaime Rodriguez-Canales ${ }^{6}$, Lorenzo Ferrando ${ }^{3}$, Nicolas Chopin ${ }^{2}$, Isabelle Ray-Coquard ${ }^{2}$, Davide Bedognetti $i^{3,7,8}$, Alberto Ballestrero ${ }^{3,5}$, Elisabetta Leo ${ }^{1, \#, *}$, Gabriele Zoppoli ${ }^{3,5, \#, *}$

\section{Affiliations:}

${ }^{1}$ Bioscience, Oncology R\&D, AstraZeneca, Cambridge, UK.

${ }^{2}$ Cancer Center Leon Bérard, Lyon, FR.

${ }^{3}$ Dipartimento di Medicina Interna e Specialità Mediche, Università degli Studi di Genova, Genova, Italy.

${ }^{4}$ Department of Integrated Surgical and Diagnostic Sciences, Università degli Studi di Genova, Genova, Italy.

${ }^{5}$ IRCCS Ospedale Policlinico San Martino, Genova, IT.

${ }^{6}$ Translational Medicine Pathology, Oncology R\&D, AstraZeneca, Gaithersburg, US.

${ }^{7}$ Cancer Research Department, Sidra Medicine, Doha, Qatar, Doha, Qatar.

${ }^{8}$ Hamad Bin Khalifa University (HBKU), Doha, Qatar.

\#These authors contributed equally to the present manuscript.

\section{*To Whom correspondence should be addressed:}

Elisabetta Leo

AstraZeneca Oncology Early Development

310 Cambridge Science Park

Milton Road

Cambridge CB4 OWG, UK

Tel +447884735447

elisabetta.leo@astrazeneca.com

Gabriele Zoppoli,

Department of Internal Medicine (DiMI), 
Viale Benedetto XV, 6, 16132 Genova, IT

Tel: +390103538667

Email: gabriele.zoppoli@unige.it

Running title: SLFN11 in ovarian cancer-immunity cycle

Key words: bimoarker, cancer-immunity cycle, high-grade serous ovarian carcinoma, immune-stroma interaction, platinum-based chemotherapy, SLFN11

\section{Abstract}

Large independent analyses on cancer cell lines followed by functional studies have identified Schlafen 11 (SLFN11), a putative DNA/RNA helicase, as the strongest predictor of sensitivity to DNA-damaging agents. However, its role as a prognostic biomarker is undefined, partially due to the lack of validated methods to score SLFN11 in human tissues. Here, we implemented a pipeline to quantify SLFN11 in human cancer samples. By analyzing a cohort of high-grade serous ovarian carcinoma specimens prior platinum-based chemotherapy treatment, we demonstrate that SLFN11 is expressed by infiltrating innate and adaptive immune cells. We show, for the first time, that SLFN11 density in both the neoplastic and microenvironmental components was independently associated with favorable outcome. Transcriptomic analyses suggested the presence of a hitherto modulation of the cancer-immunity cycle orchestrated by SLFN11. We propose SLFN11 as a dual biomarker capturing simultaneously interconnected immunological and cancercell-intrinsic functional dispositions associated with sensitivity to DNA damaging agents. 


\section{Introduction}

The putative DNA/RNA helicase Schlafen11 (SLFN11) was independently reported by us(Zoppoli et al, 2012) and others to be the top correlating transcript, amongst more than 20,000, with the response of cancer cells to DNA damaging agents (DDA) with different modes of action such as topoisomerase I (e.g. topotecan and irinotecan)(Barretina et al, 2012; Coussy et al, 2020), topoisomerase II inhibitors (e.g. epirubicin) and bulk alkylating or alkylating and crosslinking-like agents (e.g. cyclophosphamide or platinum salts, respectively)(Conteduca et al, 2020; Iwasaki et al, 2019; Stewart et al, 2017). Subsequently, a positive association between SLFN11 and sensitivity to Poly (ADP-ribose) polymerase inhibitors (PARPi) was also described(Lok et al, 2017; Murai et al, 2016; Pietanza et al, 2018; Stewart et al., 2017). After our discovery, several studies confirmed the causal role of SLFN11 in the process of cell death upon DDA challenge in cell lines(Murai et al, 2018; Murai et al, 2020), organoids(Conteduca et al., 2020) and xenografts(Coussy et al., 2020; Iwasaki et al., 2019; Stewart et al., 2017) from different tumor types. Moreover, SLFN11 has been recently studied in relation with the immune system(Mezzadra et al, 2019; Stewart et al., 2017), especially in breast cancer(Isnaldi et al, 2019), and for its potential role as an endogenous inhibitor of viral replication( $\mathrm{Li}$ et al, 2012) and translation of DNA damage response proteins(Li et al, 2018). Taken together, the available literature suggests that SLFN11 may play a so far not completely understood role in an intertwined process of cancer and immune response to DDAbased chemotherapies. Indeed, it has been shown that SLFN11 is strictly correlated with immune-related transcripts in breast cancer(Isnaldi et al., 2019), and its expression is regulated by interferon signaling in primary human cells(Li et al., 2012; Puck et al, 2015) and, possibly, also in neoplastic cells(Mezzadra et al., 2019; Stewart et al., 2017). Moreover, SLFN11 is associated with early interferon-response genes in neoplastic cells(Stewart et al., 2017), hence pointing toward an exogenous regulation of its levels by the tumor-infiltrating immune milieu. One of the human cancers whose standard-of-care (SoC) treatment relies upon DDA, and which are considered particularly sensitive to such category of chemotherapeutics, is highgrade serous ovarian carcinoma (HGSOC). HGSOC is the most common histologic subtype of ovarian cancer, accounting for three quarters of newly diagnosed cases(Lisio et al, 2019). Initial SoC treatment for advanced stage HGSOC (the most 
frequent presentation stage for this poor-prognosis disease) consists of a platinum salt-taxane chemotherapy (CT) combination regimen, interposed or preceded by surgical debulking(Lheureux et al, 2019). In spite of macroscopically complete resection (R0) and upfront chemotherapy, most HGSOC patients will eventually progress and die from their disease. In this context, several studies have shown that tumor-infiltrating lymphocytes (TILs), especially CD3+ and CD8+ TILs, may have a role as a prognostic biomarker, but their clinical utility is still unclear(Stanske et al, 2018). In this study, our main aim was to determine whether SLFN11 transcript and protein could be accurately and reproducibly measured in two different serous ovarian cancer cohorts, one internal and another one from TCGA, considering the following aspects: a) the sensitivity of HGSOC to DDA, b) the need for clinically useful prognostic biomarkers for chemotherapy treatments, c) the potential connection between SLFN11 and TILs, and, d) the potential shown by SLFN11 modulation in preclinical models. We explored how SLFN11 protein is expressed in cancer cells and their surrounding microenvironment and, most importantly, whether SLFN11 could represent a relevant prognostic biomarker to platinum-based treatment response in advanced stage HGSOC patients.

\section{Results}

1. Demographics. The clinico-pathological features of HGSOC cases selected for the present analysis, as detailed in the Methods section, are reported in Table 1 and Supplementary Table 1. The proportions of advanced stage HGSOC patients were balanced between platinum- resistant ( $P R, N=13$ ) patients, defined as progressing within six months from the end of first $C T$, and platinum-sensitive (PS, $N=15$ ) ones ( $85 \%$ and $87 \%$ respectively), as was the median number of completed cycles (seven in both groups). Median progression-free interval (PFI) was 4 months $(95 \% \mathrm{Cl}=2-$ 6) in PR patients and 11 months $(95 \% \mathrm{Cl}=9-$ not reached) in PS ones. The patients had a median age of 62.4 years $(95 \% \mathrm{Cl}=56.8-66.9)$. In the studied cohort, PR cases were on average older than PS ones (65.7 vs. 58.2 years, p-value $=0.0026)$.

\section{SLFN11 levels are precisely defined by both transcript and protein levels in} HGSOC samples. To assess SLFN11 in HGSOC cases, we evaluated both the 
transcript levels by quantitative real-time polymerase chain reaction (qRT-PCR) as $\Delta \Delta \mathrm{Ct}$, and the protein levels by immunohistochemistry (IHC) as $\mathrm{H}$-score, blindly measured by HALO (CW) in formalin-fixed, paraffin-embedded (FFPE) samples (see Supplementary Table 1 and Figure S1). Transcript and protein levels showed a strongly significant correlation $(\rho=0.52, p$-value $=0.0051$, see Figure $1 A)$. This suggests that independent methods to measure SLFN11 in FFPE tissues yield comparable results, and that assessing either SLFN11 transcript or protein are both acceptable ways to analytically quantify the tissue levels of SLFN11 gene products. We next sought to test whether SLFN11 H-scores, blindly evaluated by a trained pathologist (JR) in cancer cells, could be consistently reproduced by digital pathology software such as HALO, which allows high content imaging assessment, and can provide quantitative measures on both cancer and non-cancer cells, as well as the two combined measures ("overall H-score"). Indeed, we found that the correlation between pathology-assessed and HALO-assessed $\mathrm{H}$-scores in cancer cells was highly significant ( $\rho=0.88$, $p$-value $<0.0001$, see Figure $1 B$ ), with excellent reliability(Koo \& Li, 2016) (intraclass correlation coefficient - ICC - for agreement = 0.88 and ICC for consistency $=0.90$, see Figure $1 \mathrm{C}$ ), no relevant bias, and a very slight trend towards higher $\mathrm{H}$-scores given by HALO for higher means, as evaluated with the Bland-Altman limits of agreement method (see Figure 1D). Taken together, these results established the analytical validity of our IHC approach to SLFN11 measurement in tumor specimens.

\section{Total and intratumoral infiltrating lymphocytes contribute to SLFN11 levels}

in HGSOC. Since several studies have reported on the role of TILs in the prognosis of ovarian cancer (Goode et al, 2017; Hwang et al, 2012; Li et al, 2017; Sato et al, 2005), and SLFN11 has been shown to be expressed in primary human Tlymphocytes(Puck et al., 2015), we evaluated TIL infiltration by CD3 and CD8 staining (analysis performed by FG), both in terms of total number/mm (total CD3+ and CD8+ TILs) and as a measure of TILs in direct contact with cancer cells, without stroma interposition (intratumoral CD3+ and CD8+ TILs) in HGSOC(Stanske et al., 2018). Using HALO, we then calculated SLFN11 overall H-scores in the studied cohort, as well as $\mathrm{H}$-scores in cancer and non-cancer cells separately (see Supplementary Table 1). H-scores measured in non-cancer cells showed the 
strongest correlations with TILs, whereas the H-scores in cancer cells exhibited a non-significant negative association with TIL counts (see Figure 2A and Supplementary Table 2). In particular, non-cancer $\mathrm{H}$-score correlations with total CD3+ and CD8+ TILs were moderate at 0.41 (false discovery rate - FDR $=0.0723$, see Figure $2 \mathrm{~B}$ ) and 0.39 (FDR $=0.0852$, see Figure $2 \mathrm{C}$ ) respectively. Indeed, SLFN11 protein assessment in the clinical specimens revealed its localization in both cancer cells and stromal cells of various origins (see Figure 2D for representative images of stroma SLFN11-high and tumor SLFN11-high or SLFN11-low pictures). Taken together, these results indicate that, in addition to TILs, other cell populations contribute to SLFN11 protein levels in tumor tissues. Of interest, a moderate association between cancer and non-cancer SLFN11 levels could be observed ( $\rho=$ $0.50, \mathrm{FDR}=0.0208)$.

\section{SLFN11 in cancer and non-cancer cells independently predicts response to} platinum-based chemotherapy in HGSOC. We next sought to explore whether higher SLFN11 protein levels associate with better outcome in platinum-treated advanced stage in our HGSOC cohort. First, we evaluated the impact of SLFN11 overall $\mathrm{H}$-score, $\mathrm{H}$-score in cancer and non-cancer cells, as well as stage, age, and TIL infiltration on PFI by univariable statistics. Overall and non-cancer SLFN11 Hscores were strongly associated with a better prognosis $(\mathrm{HR}=0.50,95 \% \mathrm{Cl}=0.33-$ $0.75, \mathrm{p}$-value $=0.0009$, and $\mathrm{HR}=0.54,95 \% \mathrm{Cl}=0.36-0.81, \mathrm{p}$-value $=0.0028$ respectively). Among the other variables with possible prognostic impact assessed in our cohort, older age was associated with shorter PFI (hazard ratio - HR $=1.83$, $95 \%$ confidence interval $-95 \% \mathrm{Cl}=1.19-2.82$, $\mathrm{p}$-value $=0.0062$ ), whereas higher total CD3+ TILs were associated with longer PFI $(\mathrm{HR}=0.55,95 \% \mathrm{Cl}=0.34-0.90$, $\mathrm{p}$-value $=0.0180$ ). A borderline significant association with shorter PFI was observed for stage IVa vs. Illc cancer $(\mathrm{HR}=3.19,95 \% \mathrm{Cl}=0.89-11.46$, p-value $=0.0758)$, whereas an opposite, trend be found for higher total CD8+ TIL count (HR $=0.67$, $95 \% \mathrm{Cl}=0.41-1.09, \mathrm{p}$-value $=0.1040)$ and SLFN11 $\mathrm{H}$-score assessed in cancer cells only $(\mathrm{HR}=0.62,95 \% \mathrm{Cl}=0.38-1.02$, p-value $=0.0620)$. With an $\mathrm{H}$-score cutoff of 60 , obtained by maximizing the accuracy to classify PR versus PS cases in our cohort, overall SLFN11 protein levels had an accuracy $=0.78$, with sensitivity $=0.93$ and specificity $=0.62$ (see Figure $3 \mathrm{~A}$ ). The association of overall SLFN11 $\mathrm{H}$-score as 
a binary variable with $\mathrm{PFI}$ was indeed significant, with an $\mathrm{HR}=0.17(95 \% \mathrm{Cl} 0.06-$ 0.45 , p-value $=0.0004$, see Figure $3 \mathrm{~B}$ ). When the most significant measure of SLFN11, i.e. the overall $\mathrm{H}$-score, and the other variables with a $\mathrm{p}$-value $\leq 0.1$ were entered in a stepwise forward-backward multivariable Cox's regression model, overall SLFN11 protein levels retained their independent prognostic value (adjusted $\mathrm{HR}=0.56,95 \% \mathrm{Cl}=0.37-0.85, \mathrm{p}$-value $=0.0073)$, together with age and stage (see Figure 3C). Albeit exploratory in nature, these results were surprising in several regards. First, the independent prognostic value of overall SLFN11 H-score suggests that SLFN11 levels in both cancer and non-cancer cells may play a role in response to platinum-containing regimens in HGSOC. Indeed, dichotomized SLFN11 cancer (see Supplementary Figures 2A and 2B) and non-cancer (see Supplementary Figures 2C and 2D) levels were also prognostic by univariable analysis (even though with smaller significance than overall SLFN11, see Supplementary Table 3). Moreover, both cancer and non-cancer SLFN11 retained their independent role in stepwise multivariable models starting from the same set of variables as the one including overall SLFN11 (see Supplementary Figures $2 \mathrm{E}$ and $2 \mathrm{~F}$ and Supplementary Table 3). An extremely interesting finding is that, when overall SLFN11 or non-cancer SLFN11 are considered together with the other covariates to generate a multivariable model, CD3+ TILs lost their independent prognostic role. On the other hand, when cancer SLFN11 $\mathrm{H}$-score is used to generate a multivariable model, that measure is independently significant together with CD3+ TILs (see Supplementary Figure 2E). This observation strengthens the hypothesis that SLFN11 expressed in cancer cells, as observed in other studies, is directly linked with neoplastic cell sensitivity to alkylating agents independently of immune infiltration, whereas the prognostic relevance of non-cancer SLFN11 may instead underlie an "active" tumor immune milieu. In turn, these findings would explain why overall SLFN11 $\mathrm{H}$-score is a stronger prognostic biomarker than either cancer or non-cancer SLFN11 measured separately.

\section{SLFN11 is expressed by cells of the innate and adaptive immune system} infiltrating HGSOC. As our results indicated, SLFN11 plays a role in the response 
to platinum-based treatment in HGSOC, due to its expression in both cancer and non-cancer (immune-related) cells. Moreover, non-cancer SLFN11 H-score shows only a moderate correlation with TILs, and they retain an independent prognostic value in HGSOC, thus suggesting that other cell subpopulations contribute to the overall levels of SLFN11 in tissues. Hence, we sought to better define these populations. With this aim, we estimated cancer cellularity in a second HGSOC cohort from The Cancer Genome Atlas (TCGA N = 302 cases) with ESTIMATE(Yoshihara et al, 2013), and we inferred leukocyte subpopulations using CIBERSORTx, a well-established method for characterizing the immune cell composition of tissues from their gene expression profiles(Newman et al, 2015). We first correlated the obtained values with SLFN11 transcript levels (see Supplementary Table 4). Of interest, not only adaptive immune system cells (CD4+, CD8+ T-cells as well as B cells), but also macrophages and Natural Killer (NK) cells showed a significant association with SLFN11 (FDR < 0.05). In fact, amongst immune cell subpopulations, macrophages showed the strictest correlations with SLFN11, and such observation was transversally confirmed for validation purposes by single sample gene set enrichment analysis (sSGSEA, correlation between enrichment score for macrophages and SLFN11 $=0.27$, p-value $<0.0001$, see Supplementary Figure 3A). Moreover, publicly available RNA-sequencing data from sorted leukocyte subpopulations (GEO accession GSE60420) corroborated our in silico findings: higher SLFN11 levels were observed in monocytes, followed by NK cells, CD8+ T-cells, B- cells and CD4+ T-cells, whereas - as in our results - the lowest SLFN11 transcript could be observed in neutrophils (see Supplementary Figure 3B). Cancer cellularity showed a negative correlation with SLFN11 expression in HGSOC TCGA OVCAR ( $\rho=-0.30$, FDR < 0.0001, see Supplementary Figure 4A). This observation, independently confirmed in our cohort $(\rho=-0.42$, $p$-value $=0.0276$, see Supplementary Figure 4B), could be explained by hypothesizing that HGSOC cases with lower cancer cellularity would, in general, have higher tumor immune cell infiltration, with consequently higher SLFN11 levels. To explore this hypothesis, we performed principal component analysis (PCA), including cancer cellularity together with immune cell subpopulations correlating with SLFN11 with FDR $<0.05$ (see Figure 4A). PCA is a dimensionality reduction method enabling the identification and visualization of correlations and patterns between variables without aprioristic 
assumptions about their mutual relationships. As anticipated, all immune subpopulations are represented as lying in opposition to cancer cellularity, in particular M1 macrophages, CD4+ memory resting T-cells and CD8+ T-cells. Moreover, the SLFN11 expression vector is lying close to those of the immune subpopulations it is more closely correlated to, such as T-cells and macrophages. Overall, PCA substantiates our hypothesis that high immune infiltration is driving the negative correlation of SLFN11 with cancer cellularity and that specific immune subpopulations are closely associated with high SLFN11 expression in HGSOC.

6. SLFN11 is independently prognostic in the TCGA HGSOC data set. Finally, we validated the prognostic role of SLFN11 in TCGA HGSOC patients. To do so, we selected stage IIIc/IV cases with histological grade 3 and at least 28 days of progression-free interval (PFI - 221 cases with 157 progression events). SLFN11 was associated with $\mathrm{PFI}(\mathrm{HR}=0.68,95 \% \mathrm{Cl}=0.49-0.95$, $\mathrm{p}$-value $=0.0233$, see Figure 4B), and remained independently significant (adjusted $\mathrm{HR}=0.67,95 \% \mathrm{Cl}=$ $0.47-0.94, p$-value $=0.0222)$, together with age and specific immune cell subpopulations, in a multivariable Cox's proportional hazards model with variables selected by lasso regularization (see Figure $4 \mathrm{C}$ ). Of relevance, B and T-cell subpopulations were independently prognostic of an extended PFI, whereas monocytes, M2 (but not M1) macrophages, and activated NK cells were associated with poorer prognosis (for univariable survival analyses of immune cell subpopulation in the TCGA data set, see Supplementary Table 5). This result is in line with SLFN11 correlations with immune cell subpopulations, in that B-cells and NK resting cells were associated with higher SLFN11 transcript, while NK activated cells were not. The surprisingly negative association between NK and prognosis might be related to imbalance of different NK cell subsets associated with diverse immune-modulatory properties. Monocytes and macrophages exhibited heterogeneous behavior in regard with prognosis when assessed in a multivariable fashion. We did not, however, try to model the interactions of SLFN11 with those subpopulations, due to the interpretational complexity of results in the absence of functional experiments, as well as the limited numerosity of the TCGA HGSOC dataset for such a purpose. Likewise, we wished to avoid the overinterpretation of an exploratory analysis which 
would, anyway, be derived from in silico deconvolution methods not devoid of the potential for error propagation.

\section{SLFN11 protein localization with immune cell subpopulations is confirmed} in tonsil and HGSOC tissues. SLFN11 protein localization in a subset of cells in the immune cell compartment could be confirmed in tonsil and HGSOC (Figure 5) tissues from our cohort. In the tonsil, SLFN11 positive cells could be mainly found in the germinal center and the paracortical zone, and less in the mantle zone, of the lymphoid follicle. The germinal center is enriched in naïve and memory B-cells $(\mathrm{CD} 20+)$ and monocyte/macrophages (CD68+), whereas the paracortical zone is mostly composed of T-cells (e.g. CD3+ and CD8+) (Figure 5A and supplementary figure 5). In HGSOC tissues we could observe high SLFN11 protein in those immune cell subtypes, particularly in monocytes/macrophages (Figure 5B, C for representative HGSOCs with varied SLFN11 protein in cancer cells). However, we also noted SLFN11 in other, to be defined, stromal cell subtypes. Taken together, we confirm that SLFN11 is expressed in macrophages, TILS and B-cells, but also in other still to be defined stromal cell types in HGSOC.

\section{High SLFN11 expression is associated with immune activity signatures in} HGSOC. After demonstrating, in silico and through immunohistochemical analysis on an independent case cohort, that SLFN11 is not only expressed in cancer cells, but also in TILs, in macrophages, and other immune cell subpopulations, we wondered how SLFN11 expression in HGSOC is correlated with biologically selected, wellestablished immune signatures representing hallmarks of immune activity, such as interferon $\alpha$ and $y$ signaling and STAT1 activation(Thorsson et al, 2018) (Liberzon et al, 2015; Teschendorff et al, 2010), MHC I and MHC II upregulation(Rody et al, 2009; Rody et al, 2011), antigen presenting machinery(Senbabaoglu et al, 2016) , immune xonstant of rejection(Bedognetti et al, 2016; Hendrickx et al, 2017) and immunogenic cell death(Garg et al, 2016) . To do so, we correlated SLFN11 with those signatures in the HGSOC TCGA RNA-seq dataset ( $\mathrm{N}=302$ cases). All the aforementioned signatures showed extremely significant positive correlations with SLFN11 expression, with close reproducibility among signatures of similar meaning generated by different authors (see Figure 6). In particular, the two top correlating 
signatures with SLFN11 in HGSOC were the immunogenic cell death signature (FDR $=5.44 \times 10)$ and the interferon $\mathrm{y}$ response hallmark signature $($ FDR $=1.23 \times 10)$. Taken together, these findings are suggestive of a close, hitherto scarcely investigated link between SLFN11 and cancer immunity in HGSOC.

\section{Discussion}

Since the discovery of the human SLFN isoform SLFN11 in 2009(Bustos et al, 2009), SLFN11 has been reported to play a role in the native immune response such as viral infections and interferon(Li et al., 2012; Puck et al., 2015), as well as a potential role in adaptive immunity in cancer(Isnaldi et al., 2019; Stewart et al., 2017). In addition, work from various groups confirmed SLFN11 as being a determinant of sensitivity to a broad range of DDAs with different modes of action(Barretina et al., 2012; Conteduca et al., 2020; Coussy et al., 2020; Deng et al, 2015; Iwasaki et al., 2019; Stewart et al., 2017; Zoppoli et al., 2012), as well as PARP inhibitors(Lok et al., 2017; Murai et al., 2016; Pietanza et al., 2018; Stewart et al., 2017), in different, mainly preclinical, cancer settings. An understanding how SLFN11 modulates the response to chemotherapy in patients is of paramount importance for both basic biology and clinical viewpoints and is currently missing.

The assessment of SLFN11 as a clinical biomarker is hindered by the lack of validate algorithms to score it in human tissues. By implementing analytic pipelines in clinical material, we demonstrate that SLFN11 in both the neoplastic and microenvironmental compartments of tumor specimens modulates the response to platinum-containing regimens in HGSOC patients. Our proposed working model, based by integrating our data with current knowledge, is as follows (Fig. 7): SLFN11 expression might be controlled by endogenous (i.e. methylation) and exogenous factors (i.e., interferon gamma produced by tumor-reactive T cells). SLFN11 high cancer cells are more prone to undergo immunogenic cell death spontaneously or following platinum or other DNA damaging agent's administration(Garg et al, 2017). Accordingly, T-cells are recruited and activate the interferon signaling cascade with consequent upregulation of $\mathrm{MHC} \mathrm{I}$ and $\mathrm{MHC}$ II molecules, and induction of the cancer-immunity cycle. SLFN11 in cancer cells may directly contribute to immune system activation by inducing tumor necrosis factor- and innate immune response 
pathways(Murai et al., 2020) and interferon signaling may directly and indirectly prompt cytotoxicity in cancer cells(Mezzadra et al., 2019).

SLFN11 has been shown to predict response to DDA in different cancer models(Conteduca et al., 2020; Coussy et al., 2020; Deng et al., 2015; Iwasaki et al., 2019; Shee et al, 2019; Zoppoli et al., 2012), but to our knowledge we describe here for the first time that SLFN11 has more predictive power when its expression in the stromal non-cancer compartment is taken into account. These results are important in several regards. First, they support overall SLFN11 profiling in clinical tissues, and we provide evidence that levels of SLFN11 can be precisely measured by both transcript and protein analyses. Second, these findings point out that SLFN11, which is only expressed in humans and some primates, should be best assessed in clinical tissues, rather than xenograft- or in vitro cancer tissues, to understand its relevance in a clinical setting.

For the first time we confirm SLFN11 expression in tumor infiltrating innate (macrophages and NK cells) and adaptive immune cells (CD4+, CD3+ and CD8+ lymphocytes and B-cells). Our results support and add to recent findings, where it has been proposed that SLFN11 plays a role not only in the innate immune response such as defense mechanisms against viruses(Li et al., 2012) or damaged DNA(Mu et al, 2016; Murai et al., 2018; Murai et al., 2020), but also in adaptive immunity to combat cancer(Isnaldi et al., 2019; Stewart et al., 2017). Accordingly, Isnaldi et al. (2019) described an association of SLFN11 with TILs in breast cancer. Our results indicate high SLFN11 expression in macrophages and monocytes, in line with a previous report(Puck et al., 2015), whereas low expression was observed in neutrophils. SLFN11 protein localization was confirmed in macrophages, CD3+ and CD8+ TILs and naïve and memory B-cells in HGSOC tissues. Macrophages, when polarized to a M1 phenotype, are important to sustain a cytotoxic $\mathrm{T}$ cell response(Sica \& Mantovani, 2012). However, we also noted SLFN11 presence in other stromal cell types that remain to be identified. Moreover, as SLFN11 is expressed in several infiltrating immune cells, it holds promise as a potential biomarker for immune infiltration and for response to immunotherapy drugs, although this warrants further investigations.

In our analyses, we demonstrate that SLFN11 in cancer and immune cells is independently a predictor of response to chemotherapy in HGSOC. These 
observations are in agreement with recent findings. Accordingly, in two other studies SLFN11 has been shown to be a predictive biomarker of longer survival in chemotherapy-treated ovarian cancer patient cohorts(Shee et al., 2019; Zoppoli et al., 2012). The concept that the immune-infiltrating milieu can modulate cancer prognosis is not new: multiple studies have reported on the role of TILs in the prognosis of ovarian cancer and HGSOC(Goode et al., 2017; Hwang et al., 2012; Li et al., 2017; Sato et al., 2005) and Liu et al. (2020) expanded this notion by looking at other leukocyte subpopulations(Liu et al, 2020). In analogy with these studies, we found that SLFN11 and TILs were associated with a better prognosis in our HGSOC cohorts. Similar trends were noted for naïve B-cells and macrophages of type 1; on the contrary, macrophages of type 2 were associated with poorer prognosis. We also noted that SLFN11 is correlated with well established, biologically selected, immune signatures (Fig. 6) in HGSOC. All together, these observations hint at the potential presence of interactions between SLFN11 and the immune system in cancer.

Potential interactions between SLFN11 and the immune system were proposed in literature. For example, SLFN11 has been described to be an IFNstimulated gene in human blood mono-nuclear cells (PBMC)(Li et al., 2012) and primary human immune cells (monocytes and monocyte-derived dendritic cells)(Puck et al., 2015), and possibly also in cancer cells, fostering further investigations. Increased cancer SLFN11 (modulated by other factors like promoter demethylation(Nogales et al, 2015)), has been proposed to activate immediate early genes to stimulate interferon signaling in response to replication stress and DNA damage(Murai et al., 2020). In a different study, SLFN11 was identified as a sensitizer of tumor cells to T cell- and IFN- $\gamma$-mediated cytotoxicity.(Mezzadra et al., 2019) Accordingly, following IFN- $\gamma$ exposure SLFN11 has been shown to couple interferon- $\gamma$ receptor (IFNGR) signaling to the induction of DNA damage and cell death in tumor cells in a context-dependent fashion. Taken together, these results point at a complex interplay between SLFN11 in cancer cells and the immune system in cancer, which merits enticing further investigation.

We are aware of the limitations of our study. Among them, the retrospective nature of the analysis is unavoidable. The second limitation is the small sample size of our clinical cohort. Nevertheless, the fact that our observations are translated to a larger HGSOC cohort from TCGA, is assuring. Thirdly, even though SLFN11 
expression in different leukocyte subpopulations could be confirmed in serial sections of tonsil and HGSOC tissues, these observations remain to be further validated by a multiplexing IHC approach. Nonetheless, to our knowledge, we show here for the first time in an in silico effort and in direct measurement SLFN11 presence and localization in tumor- and the tumor infiltrating milieu, in HGSOC patients.

In summary, the current study shows that SLFN11 in both cancer cells and a multitude of immune cells and potentially other (to be defined) cell types is associated with a better survival of HGSOC patients treated with platinum-containing regimens. Our findings add important information on the action of SLFN11 beyond its recently described role hence, we propose SLFN11 as a dual biomarker capturing simultaneously interconnected immunological and cancer-cell-intrinsic functional dispositions associated with sensitivity to DNA damaging agents.

\section{Patients and methods}

Study design. The objective of this study was to identify the association of SLFN11 with immune infiltration and prognosis in advanced high-grade serous ovarian carcinoma (HGSOC) patients undergoing neoadjuvant chemotherapy containing platinum. SLFN11 transcript was evaluated by RT-PCR and SLFN11 protein and immune infiltration localization by IHC. SLFN11 transcript and protein from noncancer and cancer cells were correlated with progression-free interval (PFI, defined as the time elapsing from the end of first treatment to clinical and/or radiological progression) to assess the value of SLFN11 as a biomarker of response to first-line platinum-containing chemotherapy regimens in HGSOC. Results were validated with a larger HGSOC cohort from TCGA.

Patients. Patients receiving a diagnosis of HGSOC, treated with neoadjuvant platinum-based chemotherapy at Léon Bérard Cancer Center, Lyon FR, from January 2008 to June 2014, and meeting the following criteria, were retrospectively included, in a consecutive fashion, for the reported analyses: written informed consent for biobanking and use of samples for research purposes according to the Hosting Institution, histologically confirmed HGSOC (grade 3 according to the AJCC TNM stage(Amin et al, 2017), radiological and/or surgical classification as stage 
IIIc/IVa (FIGO classification)(Berek et al, 2018) at diagnosis, Eastern Cooperative Group (ECOG) performance status 0-1 at diagnosis, postmenopausal status at diagnosis, platinum-based neoadjuvant treatment at diagnosis followed by surgery, availability of a formalin-fixed, paraffin-embedded (FFPE) pre-chemotherapy tumor block from diagnostic biopsy, availability of clinical information concerning treatment and response duration and disease status at the time of sample collection. Cases were excluded: if they had primary debulking surgery followed by chemotherapy, if they were stage $\mathrm{IVb}$, if they had received a previous or concomitant diagnosis of neoplasia (with the exclusion of carcinoma in situ of the cervix or skin basalioma) or if patients had received previous chemotherapies for any reason. Patients were divided into platinum-sensitive and platinum-resistant groups as previously described(Friedlander et al, 2011; Wilson et al, 2017). The presented research was conducted according to the ethical considerations and in compliance with the principles of the Declaration of Helsinki, approved by Regione Liguria Ethics Committee with registration number 347/2018 (approved 19/06/2019).

RT-PCR. SLFN11 transcript quantification by q-RT-PCR was performed as previously described(Garutia et al, 2014). In brief, three membrane glass slides (PEN Membrane Glass slides, Arcturus ${ }^{\circledR}$ Bioscience Inc. CA, USA), loaded with 8$\mu \mathrm{m}$ thick sections were cut from FFPE-embedded pre-treatment diagnostic biopsies. One section of each sample was hematoxylin/eosin-stained and examined to assess tumor cellularity. Samples with a $<70 \%$ cellularity were subjected to microdissection. Upon RNA extraction and retro-transcription, RNA was quantified by Q-RT-PCR using a SLFN11-specific InvitrogenTM TaqMan® assay (Invitrogen Inc. CA, USA) on an Applied Biosystems Inc. HT-7900 instrument. Samples were analysed in triplicate, using RPLP0, GAPDH and GUS as housekeeping (HK) genes. The mean PCR cycle thresholds $(\mathrm{Ct})$ of the three HK genes was subtracted from the Ct value of SLFN11 for each sample, expressed as $\log _{2}(\Delta \mathrm{Ct})$ and, in turn, the median of $\Delta$ Cts from the dataset was subtracted from $\Delta \mathrm{Cts}$ of single samples and inverted, to obtain a normally distributed, zero-centered semi-quantitative value for each sample ($\Delta \Delta \mathrm{Ct}$ ), as previously described(Barretina et al., 2012). 
RNA-sequencing profiling of human leukocyte subpopulations. RNAsequencing results for SLFN11 in sorted leukocyte subpopulations from patients with immune-associated diseases, as further described by Linsley PS et al(Linsley et al, 2014), were obtained from the Gene Expression Omnibus (GEO, accession number GSE60424).

\section{Immunohistochemistry (IHC) and image analysis for SLFN11, CD3, CD8, CD20} and CD68. SLFN11 IHC was performed on $4 \mu \mathrm{M}$ thick sections of formalin fixed paraffin embedded (FFPE) tissues and carried out on Bond RX (Leica Microsystem) using ER1 ( $\mathrm{pH} 6$, Leica) antigen retrieval. Slides were stained with primary rabbit polyclonal anti-SLFN11 antibody (Abcam ab121731) at $2.5 \mu \mathrm{g} / \mathrm{ml}$. Detection was performed with anti-rabbit poly-HRP-IGG, DAB refine and DAB enhancer (Leica, polymer refine detection kit, Leica). Digital slide images were acquired with the Aperio AT2 scanner (Leica) using a 20x objective. A HALO (Indica Labs) cytonuclear image analysis algorithm was optimized and run alongside different tissue classifiers and annotations, to capture the percentage of cancer, non-cancer and overall (cancer + non-cancer) nuclei with strong $(3+)$, moderate $(2+)$, weak $(1+)$ or negative staining to calculate SLFN11 H-scores as $[(\% 1+$ cells $)+(\% 2+$ cells * 2$)+(\% 3+$ cells * 3)] in each sample. Samples were H\&E stained to identify cancer cells. The same algorithm was used across all specimens and the analysis was blindly performed. All samples were in addition manually evaluated by a pathologist for cancer SLFN11 Hscores. CD3 and CD8 IHC was performed on full thick sections of FFPE tissues and carried out on Ventana Benchmark Ultra (Ventana Medical Systems) using heatbased antigen retrieval. Slides were stained with primary rabbit monoclonal anti-CD3 antibody (clone 2GV6 at $2.5 \mu \mathrm{g} / \mathrm{ml}$ ) and primary rabbit monoclonal anti-CD8 antibody (clone SP57 at $2.0 \mu \mathrm{g} / \mathrm{ml}$ ), both from Ventana Medical Systems. Slides were evaluated by a pathologist for total and intratumoral CD3 and CD8 by calculating mean CD3 and CD8 values from three high power field regions per sample. To confirm spatial resolution of SLFN11 protein in immune infiltrating cells, $4 \mu \mathrm{m}$ serial FFPE sections of human tonsil tissue or HGSOC specimens were taken. Sections were IHC stained for CD3, isotope control, SLFN11, CD8, CD20 and CD68 with BOND RX using ER1 (CD8 and CD20) or ER2 (CD3, IGG, SLFN11 and CD68) antigen retrieval. Primary antibodies used were as following: anti-SLFN11 as 
described above, anti-isotype control (ab172730, Abcam, at $2.5 \mu \mathrm{g} / \mathrm{ml}$ ), anti-CD3 (clone 2GV6, Roche, at $0.4 \mu \mathrm{g} / \mathrm{ml}$ ), anti-CD8 (clone C8/144 B, Dako, at $157 \mu \mathrm{g} / \mathrm{ml}$ ), anti-CD20 (clone L26, Abcam, at $33.3 \mu \mathrm{g} / \mathrm{ml}$ ) and anti-CD68 antibodies (clone PGM1, Dako, at $0.3 \mu \mathrm{g} / \mathrm{ml}$ ). Detection was performed with poly-HRP-IGG, DAB refine and $\mathrm{DAB}$ enhancer (polymer refine detection kit, Leica). Digital slide images were acquired with the Aperio AT2 scanner (Leica) using a 20x or 40x objective.

Statistical analyses. Correlations between continuous variables were calculated using the Spearman's rank coefficient and represented using scatter plots (package CNtu(Desmedt et al, 2016)), whereas differences in continuous distributions were calculated using the Wilcoxon test without continuity correction. Intraclass correlation coefficients (ICCS) were calculated to assess the consistency and agreement of IHC assessments (package psy(Shrout \& Fleiss, 1979)), and visually inspected for bias and trend using dot plots and Bland-Altman plots. Correlation matrices and correlograms of IHC and TILs were generated using the package corrgram(Friendly, 2002), and multiple tests for association were adjusted using the BenjaminiHochberg method. Univariable Cox's proportional hazards regression models were used for associations with $\mathrm{PFI}$, after log2 transformation and scaling of continuous measures. $\mathrm{HR}, 95 \% \mathrm{Cl}$ and $\mathrm{p}$-values according to the Wald statistics were reported. For multivariable Cox's regression, variables with a $p$-value $<0.1$ were entered in a stepwise forward-backward model minimizing the Akaike Information Criterion (package MASS(Venables \& Ripley, 2002)). "Optimal” (quoted because considerable as such only in the examined case set) cutoffs to dichotomize continuous variables were obtained using binary class labels (i.e. NR vs PR patients) and maximizing the accuracy to correctly classify those classes with the package cutpointr(Thiele, 2019). Forest plots representing adjusted $\mathrm{HR}$ and $95 \% \mathrm{Cls}$ were generated with the package survminer(Kassambara et al, 2019). To estimate the relative abundances of cell types in gene expression mixtures from the Cancer Genome Atlas high-grade ovarian cancer data (OVCAR) - processed and normalized as described in Roelands $\mathrm{J}$, et al.,(Roelands et al, 2019) the count matrix was analyzed using CIBERSORTx(Newman et al, 2019) on the dedicated web tool available at the URL https://cibersortx.stanford.edu/. The following parameters were set for the analysis: impute cell fractions, signature file LM22.update-gene-symbols.txt, batch correction 
enabled, batch correction mode B-mode, disable quantile normalization true, run mode absolute, permutation number 1,000. The job was performed on March 6, 2020. To derive cancer cellularity, we used ESTIMATE(Yoshihara et al., 2013) with default parameters, after log2 transformation and offsetting count data by a value = 1. Single sample gene set enrichment analysis for selected immune phenotypes, gene, and hallmark immune signatures was obtained as previously described(Bindea et al, 2013; Liberzon et al., 2015; Roelands et al., 2019; Thorsson et al., 2018). For univariable analysis of correlations between SLFN11 expression and cell fractions, we calculated the Spearman's correlation coefficient. P-values were adjusted for multiple testing using the Benjamini-Hochberg method. For multivariate analysis of CIBERSORTx cell fractions and SLFN11 expression in the HGSOC TCGA dataset, we included variables with FDR $<0.05$ for correlation with SLFN11, including cancer cellularity, after normalizing vectors as follows: we first removed near-zero variance variables (caret package(Kuhn et al, 2020)), then we pseudo-normalized data using the Tukey's ladder of power transformation method (rcompanion package(Mangiafico, 2020)), finally we centered and scaled them. The relationship between variables was represented using a variable correlation plot, with SLFN11 expression as a supplementary quantitative variable(Lê et al, 2008). For survival analyses, we selected TCGA OVCAR (HGSOC) cases with the following characteristics: stage IIIc/IV, histologic grade III, and with PFI > 28 days. SLFN11 was considered "high" when in the top two tertiles of expression, and "low" otherwise. Univariable Cox's regression and Kaplan-Meier curves were used as described above. For multivariable Cox's regression, we first transformed bimodal CIBERSORTx cell fractions into binary factors ("present" vs. "absent") if the Hartigan's dip test for unimodality was rejected with $p$-value $<0.01$. We then performed feature selection starting from stage, age, SLFN11 transcript, and CIBERSORTx variables. To do so, we fitted the Cox's regression model by regularizing it with a lasso penalty, using the package glmnet(Friedman et al, 2010) with default options ( $\alpha=1,10$-fold cross-validation), and iterating it 1,000 times to obtain the minimum average error of the regularization parameter lambda for variable selection. Finally, selected variables were entered in a Cox's multiple regression model to report $\mathrm{HR}$ point estimates and $95 \% \mathrm{Cl}$. The dendrogram of similarity between immunologic signatures was built through hierarchical clustering 
using the Ward's criterion agglomeration method and Euclidean distance between variables(Murtagh \& Legendre, 2014).

Power considerations. The sample size for the present study was meant to identify a clinically significant difference in SLFN11 expression between platinum-resistant (PR) patients, defined as relapsing within six months from the end of chemotherapy, and platinum-sensitive (PS) ones (i.e. relapsing beyond six months from the end of treatment). The suggested size of the collected cohort was based on our previous findings of a very significant hazard ratio in terms of overall survival between "SLFN11-high" and "SLFN11-low" HGSOC patients, with SLFN11 levels deemed so if being above or below the median for the considered cohort(Zoppoli et al., 2012). The required sample size would be of 24 patients, equally allocated in two groups of 12 PR and 12 PS ones, assuming a proportion of "SLFN11-high" patients of $10 \%$ in the first group and $70 \%$ in the second groups, with two-tailed $\alpha=.05$ and $1-\beta=.9$ (z test family, $G^{*}$ Power 3.1.4). Assuming that $20 \%$ of samples could not be analyzed due to failure in sample processing or testing, it was estimated that 28 samples were a sufficient number needed to test the aforementioned hypothesis.

Acknowledgements. Maria Udriste, Sophie E. Willis and Elisabeth Wiseman are thanked for generating sections of FFPE samples for IHC analyses. G.Z. wishes to thank Dr. P. Blandini for his precious insights throughout the presented research.

Author contributions. Conceptualisation \& design: C.W., D.B., G.Z; Methodology, software \& data collection: C.W., F.D., A.G., F.G., J.R., L.R., D.B., G.Z.; Writing original draft and visualisation: C.W., D.F., D.B., E.L., G.Z.; Writing -review \& editing: all authors; Supervision: E.L., G.Z.; Project administration: N.C., I.RC., E.L., G.Z; Funding acquisition: E.L., G.Z. Competing interests. E.L. is a full-time employee of AstraZeneca. C.W. is a PostDoc Fellow of the AstraZeneca PostDoc program.

Financial support: We thank AstraZeneca for PostDoc Fellowship funding for this project. G.Z. is supported by an AIRC IG grant ID 21761 and by intramural research funds from Università degli Studi di Genova. 


\section{References}

Amin MB, Edge S, Greene F, Byrd DR, Brookland RK, Washington MK, Gershenwald JE, Compton CC, Hess KR, Sullivan DC et al (2017) American Joint Committee on Cancer. Ovary, Fallopian Tube, and Primary Peritoneal Carcinoma. In: AJCC Cancer Staging Manual 8th ed Chicago, IL: American College of Surgeons, pp. 689-698. New York: Springer

Barretina J, Caponigro G, Stransky N, Venkatesan K, Margolin AA, Kim S, Wilson CJ, Lehar J, Kryukov GV, Sonkin D et al (2012) The Cancer Cell Line Encyclopedia enables predictive modelling of anticancer drug sensitivity. Nature 483: 603-607

Bedognetti D, Hendrickx W, Ceccarelli M, DMiller L, Seliger B (2016) Disentangling the relationship between tumor genetic programs and immune responsiveness. Current Opinion in Immunology 39: 150-158

Berek JS, Kehoe ST, Kumar L, Friedlander M (2018) Cancer of the ovary, fallopian tube, and peritoneum. Int J Gynaecol Obstet 143 Suppl 2: 59-78

Bindea G, Mlecnik B, Tosolini M, Kirilovsky A, Waldner M, Obenauf AC, Angell H, Fredriksen T, Lafontaine L, Berger A et al (2013) Spatiotemporal dynamics of intratumoral immune cells reveal the immune landscape in human cancer. Immunity 39: 782-795

Bustos O, Naik S, Ayers G, Casola C, Perez-Lamigueiro MA, Chippindale PT, Pritham EJ, de la Casa-Esperón E (2009) Evolution of the Schlafen genes, a gene family associated with embryonic lethality, meiotic drive, immune processes and orthopoxvirus virulence. Gene 447: 1-11

Conteduca V, Ku SY, Puca L, Slade M, Fernandez L, Hess J, Bareja R, Vlachostergios PJ, Sigouros M, Mosquera JM et al (2020) SLFN11 expression in advanced prostate cancer and response to platinum-based chemotherapy. $\mathrm{Mol}$ Cancer Ther

Coussy F, El-Botty R, Chateau-Joubert S, Dahmani A, Montaudon E, Leboucher S, Morisset L, Painsec P, Sourd L, Huguet L et al (2020) BRCAness, SLFN11, and RB1 loss predict response to topoisomerase I inhibitors in triplenegative breast cancers. Sci Transl Med 12

Deng Y, Cai Y, Huang Y, Yang Z, Bai Y, Liu Y, Deng X, Wang J (2015) High SLFN11 expression predicts better survival for patients with KRAS exon 2 wild type colorectal cancer after treated with adjuvant oxaliplatin-based treatment. BMC Cancer 15: 833

Desmedt C, Zoppoli G, Gundem G, Pruneri G, Larsimont D, Fornili M, Fumagalli D, Brown D, Rothe F, Vincent D et al (2016) Genomic Characterization of Primary Invasive Lobular Breast Cancer. J Clin Oncol 34: 1872-1881

Friedlander M, Trimble E, Tinker A, Alberts D, Avall-Lundqvist E, Brady M, Harter P, Pignata S, Pujade- Lauraine E, Sehouli J et al (2011) Clinical Trials in Recurrent Ovarian Cancer. International Journal of Gynecologic Cancer 21

Friedman J, Hastie T, Tibshirani R (2010) Regularization Paths for Generalized Linear Models via Coordinate Descent. Journal of Statistical Software 33

Friendly M (2002) Corrgrams. The American Statistician 56

Garg AD, De Ruysscher D, Agostinis P (2016) Immunological metagene signatures derived from immunogenic cancer cell death associate with improved survival of patients with lung, breast or ovarian malignancies: A large-scale metaanalysis. Oncoimmunology 5: e1069938 
Garg AD, More S, Rufo N, Mece O, Sassano ML, Agostinis P, Zitvogel L, Kroemer G, Galluzzi L (2017) Trial watch: Immunogenic cell death induction by anticancer chemotherapeutics. Oncoimmunology 6: e1386829

Garutia A, Roccoa I, Cirmena G, Chiaramondia M, Baccini P, Calabrese M, Palermo C, Friedman D, Zoppoli G, Ballestreroa A (2014) Quantitative Real Time PCR assessment of hormonal receptors and HER2 status on fine-needle aspiration pre-operatory specimens from a prospectively accrued cohort of women with suspect breast malignant lesions. Gynecologic Oncology 132: 389-396

Goode EL, Block MS, Kalli KR, Vierkant RA, Chen W, Fogarty ZC, GentryMaharaj A, Toloczko A, Hein A, Bouligny AL et al (2017) Dose-Response Association of CD8+ Tumor-Infiltrating Lymphocytes and Survival Time in HighGrade Serous Ovarian Cancer. JAMA Oncol 3: e173290

Hendrickx W, Simeone I, Anjum S, Mokrab Y, Bertucci F, Finetti P, Curigliano G, Seliger B, Cerulo L, Tomei S et al (2017) Identification of genetic determinants of breast cancer immune phenotypes by integrative genome-scale analysis. Oncoimmunology 6: e1253654

Hwang WT, Adams SF, Tahirovic E, Hagemann IS, Coukos G (2012) Prognostic significance of tumor-infiltrating $T$ cells in ovarian cancer: a metaanalysis. Gynecol Oncol 124: 192-198

Isnaldi E, Ferraioli D, Ferrando L, Brohee S, Ferrando F, Fregatti $P$, Bedognetti D, Ballestrero A, Zoppoli G (2019) Schlafen-11 expression is associated with immune signatures and basal-like phenotype in breast cancer. Breast Cancer Res Treat

Iwasaki J, Komori T, Nakagawa F, Nagase H, Uchida J, Matsuo K, Uto Y (2019) Schlafen11 Expression Is Associated With the Antitumor Activity of Trabectedin in Human Sarcoma Cell Lines. Anticancer Research 39: 3553-3563

Kassambara A, Kosinski M, Biecek P, 2019. Survminer: Drawing Survival Curves using 'ggplot2', R package version 0.4.6. ed.

Koo TK, Li MY (2016) A Guideline of Selecting and Reporting Intraclass Correlation Coefficients for Reliability Research. Journal of Chiropractic Medicine 15: 155-163

Kuhn M, Wing J, Weston S, Williams A, Keefer C, Engelhardt A, Cooper T, Mayer Z, Kenkel B, R Core Team et al, 2020. Package 'caret': Classification and Regression Training, R-package version 6.0-86 ed., p. Misc functions for training and plotting classification and

regression models.

Lê S, Josse J, Husson F (2008) FactoMineR: An R Package for Multivariate Analysis. Journal of Statistical Software 25

Lheureux S, Gourley C, Vergote I, Oza AM (2019) Epithelial ovarian cancer. The Lancet 393: 1240-1253

Li J, Wang J, Chen R, Bai Y, Lu X (2017) The prognostic value of tumorinfiltrating T lymphocytes in ovarian cancer. Oncotarget 8: 15621-15631

Li M, Kao E, Gao X, Sandig H, Limmer K, Pavon-Eternod M, Jones TE, Landry S, Pan T, Weitzman MD et al (2012) Codon-usage-based inhibition of HIV protein synthesis by human schlafen 11. Nature 491: 125-128

Li M, Kao E, Malone D, Gao X, Wang JYJ, David M (2018) DNA damageinduced cell death relies on SLFN11-dependent cleavage of distinct type II tRNAs. Nat Struct Mol Biol 25: 1047-1058 
Liberzon A, Birger C, Thorvaldsdottir H, Ghandi M, Mesirov JP, Tamayo P (2015) The Molecular Signatures Database (MSigDB) hallmark gene set collection. Cell Syst 1: 417-425

Linsley PS, Speake C, Whalen E, Chaussabel D (2014) Copy number loss of the interferon gene cluster in melanomas is linked to reduced $T$ cell infiltrate and poor patient prognosis. PLoS One 9: e109760

Lisio MA, Fu L, Goyeneche A, Gao ZH, Telleria C (2019) High-Grade Serous Ovarian Cancer: Basic Sciences, Clinical and Therapeutic Standpoints. Int J Mol Sci 20

Liu R, Hu R, Zeng Y, Zhang W, Zhou HH (2020) Tumour immune cell infiltration and survival after platinum-based chemotherapy in high-grade serous ovarian cancer subtypes: A gene expression-based computational study. EBioMedicine 51: 102602

Lok BH, Gardner EE, Schneeberger VE, Ni A, Desmeules P, Rekhtman N, de Stanchina E, Teicher BA, Riaz N, Powell SN et al (2017) PARP Inhibitor Activity Correlates with SLFN11 Expression and Demonstrates Synergy with Temozolomide in Small Cell Lung Cancer. Clinical Cancer Research 23: 523-535

Mangiafico S, 2020. Package 'rcompanion': Functions to Support Extension Education Program Evaluation.

Mezzadra R, de Bruijn M, Jae LT, Gomez-Eerland R, Duursma A, Scheeren FA, Brummelkamp TR, Schumacher TN (2019) SLFN11 can sensitize tumor cells towards IFN-gamma-mediated T cell killing. PLoS One 14: e0212053

Mu Y, Lou J, Srivastava M, Zhao B, Feng XH, Liu T, Chen J, Huang J (2016) SLFN11 inhibits checkpoint maintenance and homologous recombination repair. EMBO Rep 17: 94-109

Murai J, Feng Y, Yu K, Ru Y, Tang S, Shen Y, Y. P (2016) Resistance to PARP inhibitors by SLFN11 inactivation can be overcome by ATR inhibition. Oncotarget 7: 76534-76550

Murai J, Tang SW, Leo E, Baechler SA, Redon CE, Zhang H, Al Abo M, Rajapakse VN, Nakamura E, Jenkins LMM et al (2018) SLFN11 Blocks Stressed Replication Forks Independently of ATR. Mol Cell 69: 371-384 e376

Murai J, Zhang H, Pongor L, Tang S-W, Jo U, Moribe F, Ma Y, Tomita M, Pommier Y (2020) Chromatin Remodeling and Immediate Early Gene Activation by SLFN11 in Response to Replication Stress. Cell Reports 30: 4137-4151.e4136

Murtagh F, Legendre P (2014) Ward's Hierarchical Agglomerative Clustering Method: Which Algorithms Implement Ward's Criterion? Journal of Classification 31: 274-295

Newman AM, Liu CL, Green MR, Gentles AJ, Feng W, Xu Y, Hoang CD, Diehn M, Alizadeh AA (2015) Robust enumeration of cell subsets from tissue expression profiles. Nat Methods 12: 453-457

Newman AM, Steen CB, Liu CL, Gentles AJ, Chaudhuri AA, Scherer F, Khodadoust MS, Esfahani MS, Luca BA, Steiner D et al (2019) Determining cell type abundance and expression from bulk tissues with digital cytometry. Nat Biotechnol 37: 773-782

Nogales V, Moutinho C, Pommier Y, Reinhold C, Varma S, Moran S, Esteller $M$ (2015) Epigenetic inactivation of the putative DNA/RNA helicase SLFN11 in human cancer confers resistance to platinum drugs. Oncotarget 7: 3084-3097

Pietanza M, Waqar S, Krug L, Dowlati A, Hann L, Chiappori A, Owonikoko T, Woo K, Cardnell R, Fujimoto J et al (2018) Randomized, Double-Blind, Phase II Study ofTemozolomide in Combination With Either Veliparib or Placebo in Patients 
With Relapsed-Sensitive or Refractory Small-Cell Lung Cancer. Journal of Clinical Oncology 36: 2386-2394

Puck A, Aigner R, Modak M, Cejka P, Blaas D, Stöckl J (2015) Expression and regulation of Schlafen (SLFN) family members in primary human monocytes, monocyte-derived dendritic cells and T cells. Results in Immunology 5: 23-32

Rody A, Holtrich U, Pusztai L, Liedtke C, Gaetje R, Ruckhaeberle E, Solbach C, Hanker L, Ahr A, Metzler D et al (2009) T-cell metagene predicts a favorable prognosis in estrogen receptor-negative and HER2-positive breast cancers. Breast Cancer Res 11: R15

Rody A, Karn T, Liedtke C, Pusztai L, Ruckhaeberle E, Hanker L, Gaetje R, Solbach C, Ahr A, Metzler D et al (2011) A clinically relevant gene signature in triple negative and basal-like breast cancer. Breast Cancer Res 13: R97

Roelands J, Hendrickx W, Mall R, Saad M, Halliwill K, Zoppoli G, Curigliano G, Rinchai D, Decock J, Delogu LG et al (2019) Genomic landscape of tumor-host interactions with differential prognostic and predictive connotation. bioRxiv doi:101101/546069

Sato E, Olson SH, Ahn J, Bundy B, Nishikawa H, Qian F, Jungbluth AA, Frosina D, Gnjatic S, Ambrosone C et al (2005) Intraepithelial CD8+ tumor-infiltrating lymphocytes and a high CD8+/regulatory $T$ cell ratio are associated with favorable prognosis in ovarian cancer. PNAS 102: 18538-18543

Senbabaoglu Y, Sumer SO, Sanchez-Vega F, Bemis D, Ciriello G, Schultz N, Sander C (2016) A Multi-Method Approach for Proteomic Network Inference in 11 Human Cancers. PLoS Comput Biol 12: e1004765

Shee K, Wells JD, Jiang A, Miller TW (2019) Integrated pan-cancer gene expression and drug sensitivity analysis reveals SLFN11 mRNA as a solid tumor biomarker predictive of sensitivity to DNA-damaging chemotherapy. PLoS One 14: e0224267

Shrout PE, Fleiss JL (1979) Intraclass correlations: uses in assessing rater reliability. Psychological bulletin 86: 420-428

Sica A, Mantovani A (2012) Macrophage plasticity and polarization: in vivo veritas. J Clin Invest 122: 787-795

Stanske M, Wienert S, Castillo-Tong DC, Kreuzinger C, Vergote I, Lambrechts S, Gabra H, Gourley C, Ganapathi RN, Kolaschinski I et al (2018) Dynamics of the Intratumoral Immune Response during Progression of High-Grade Serous Ovarian Cancer. Neoplasia 20: 280-288

Stewart C, Tong P, Cardnell J, Sen T, Li L, Gay M, Masrorpour F, Fan Y, Bara $\mathrm{O}$, Feng $\mathrm{Y}$ et al (2017) Dynamic variations in epithelial-to-mesenchymal transition (EMT), ATM, and SLFN11 govern response to PARP inhibitors and cisplatin in small cell lung cancer. Oncotarget 8: 28575-28587

Teschendorff AE, Gomez S, Arenas A, El-Ashry D, Schmidt M, Gehrmann M, Caldas C (2010) Improved prognostic classification of breast cancer defined by antagonistic activation patterns of immune response pathway modules. BMC Cancer 10

Thiele C, 2019. Cutpointr: Determine and Evaluate Optimal Cutpoints in Binary Classification Tasks, R package version 1.0.1. ed.

Thorsson V, Gibbs DL, Brown SD, Wolf D, Bortone DS, Ou Yang TH, PortaPardo E, Gao GF, Plaisier CL, Eddy JA et al (2018) The Immune Landscape of Cancer. Immunity 48: 812-830 e814

Venables WN, Ripley BD (2002) Modern Applied Statistics with S, Fourth edition. Springer, New York 
Wilson MK, Pujade-Lauraine E, Aoki D, Mirza MR, Lorusso D, Oza AM, du Bois A, Vergote I, Reuss A, Bacon M et al (2017) Fifth Ovarian Cancer Consensus Conference of the Gynecologic Cancer InterGroup: recurrent disease. Annals of Oncology 28: 727-732

Yoshihara K, Shahmoradgoli M, Martinez E, Vegesna R, Kim H, TorresGarcia W, Trevino V, Shen H, Laird PW, Levine DA et al (2013) Inferring tumour purity and stromal and immune cell admixture from expression data. Nat Commun 4: 2612

Zoppoli G, Regairaz M, Leo E, Reinhold WC, Varma S, Ballestrero A, Doroshow JH, Pommier Y (2012) Putative DNA/RNA helicase Schlafen-11 (SLFN11) sensitizes cancer cells to DNA-damaging agents. Proc Natl Acad Sci U S A 109: $15030-15035$ 


\section{Figure legends:}

Figure 1: SLFN11 transcript and protein levels in HGSOC. Panel A: Scatterplot representing SLFN11 transcript by qRT-PCR as $-\triangle \Delta C t$ ( $y$ axis) as a function of its protein assessment by $\mathrm{IHC}$ as $\mathrm{H}$-score ( $x$ axis) in non-cancer and cancer nuclear cells from HGSOC specimens; $\rho$ is the Spearman's correlation coefficient, the least squares regression is represented by the red line, whereas dots are measurements of SLFN11 by qRT-PCR and IHC in individual samples. Panel B: Scatterplot representing SLFN11 protein levels in HGSOC cancer cells. $X$ axis: pathologist's assessment; $y$ axis: H-score measured by HALO Digital Pathology (DP) software.

Panel C: Dot plot illustrating cancer-cell $\mathrm{H}$-scores in individual samples ( $y$ axis), ordered by increasing DP-assigned values ( $x$ axis), highlighting the excellent consistency of intraclass correlation coefficients (ICC) between the two methods. Each dot represents a score assigned by either the DP software (HALO) or the pathologist performing the assessment. Panel D: Bland-Altman plot displaying the difference between HALO and pathologist's H-scores for cancer cells ( $y$ axis) by the increasing mean of value couples for individual samples ( $x$ axis). All points lie within 1.96 standard deviations (SD - dotted green horizontal lines) from the mean difference (dashed horizontal black line), indicating no relevant bias between raters, and an insignificant trend toward higher $\mathrm{H}$-scores for HALO as the mean values increase. The red line represents a smoothed regression (loess) fit of the actual mean scores.

Figure 2: SLFN11 protein levels in HGSOC and their correlation with TILs. Panel A: Correlogram of TILs and SLFN11 H-scores, assessed in overall, cancer and non-cancer cells. In the lower triangle of the graph, boxes represent pairwise correlations colored by direction (blue for negative correlations and red for positive ones) and strength (intensity of shading) of the correlation itself. In the upper triangle, circles use the same scaled colors, but fill an area proportional to the absolute value of the correlation, and are filled clockwise for positive values, anticlockwise for negative values. Panels $\boldsymbol{B}$ and $\boldsymbol{C}$ : Scatterplots representing total CD3+ cells - panel B - and total CD8+ cells - panel C - ( $y$ axes, cells $/ \mathrm{mm}$ ) as a function of SLFN11 $\mathrm{H}$-score in non-cancer cells ( $x$ axis); $\rho$ is the Spearman's correlation coefficient, the least squares regression are represented by the red lines, whereas 
dots are measurements of immune cell counts by $\mathrm{H}$-scores in individual samples.

Panel D: Representative images of SLFN11 IHC in HGSOC specimens. Left, stroma SLFN11 high and tumor SLFN11 low and right, stroma and tumor SLFN11 high, for the indicated cancers. The insets highlight nuclear SLFN11 protein localization in tumor cells and different stromal cell subtypes. Scale bars, $50 \mu \mathrm{m}$. The insets show a $3 x$ magnification of the representative image.

Figure 3: SLFN11 protein levels measured in cancer and non-cancer cells are independently prognostic in HGSOC. Panel A: Waterfall plot showing SLFN11 overall protein levels (i.e., measured in cancer and non-cancer cells) in individual cases, colored by platinum sensitivity: SLFN11 protein is reported as $\mathrm{H}$-score (y axis), whereas cases are reported by increasing values ( $x$ axis) and colored in red if platinum-refractory (PR) or light blue if non-refractory (NR). Panel B: Kaplan-Meier plot showing progression-free interval (PFI) stratified by SLFN11 overall protein levels ("high" if H-score > 60, "low" if < 60). The progressed fraction of patients ( $y$ axis) is plotted against time expressed in months from the end of first-line chemotherapy, censored at 24 months ( $x$ axis). Numbers at risk are reported below the plot. P-value in the bottom left of the plot is from the Wald statistics for the univariable Cox's regression. Panel $\boldsymbol{C}$ : Forest plot of hazard ratios (x axis, in log scale) for variables retained in the final multiple Cox's regression model. Point HR estimates are reported below each variable together with 95\% confidence intervals $(95 \% \mathrm{Cl})$ in parentheses, whereas adjusted p-values for each variable are on the right side of the plot. Filled black squares represent $\mathrm{HR}$ estimates, with relative $95 \% \mathrm{Cl}$ shown as horizontal lines with brackets.

Figure 4: SLFN11, immune cell subpopulations and prognosis in the TCGA serous ovarian carcinoma dataset. Panel A: Variable correlation plot of the principal component analysis including SLFN11 transcript, cancer cellularity, and CIBERSORTx immune cell subpopulations significant by univariable correlation at FDR $<0.05$. The two axes represent the first principal components explaining the greatest fraction of the variance of the analysed dataset, with percent of explained variability in parentheses. The relative position of the variables towards each other explains their relative correlation, whereas their distance from the intersect accounts 
for their contribution to the components. SLFN11 is represented with a thick red arrow for sake of clarity. Panel B: Kaplan-Meier plot showing progression-free interval (PFI) stratified by SLFN11 transcript ("high" if above the lower tertile of expression in the dataset, "low" if below). The progressed fraction of patients ( $y$ axis) is plotted against time expressed in months from the end of first-line chemotherapy, censored at 60 months ( $x$ axis). Numbers at risk are reported below the plot. P-value in the bottom left of the plot is from the Wald statistics for the univariable Cox's regression. Panel C: Forest plot of hazard ratios ( $x$ axis, in log scale) for variables retained in the lasso-selected multiple Cox's regression model. Point HR estimates are reported below each variable together with $95 \%$ confidence intervals $(95 \% \mathrm{Cl})$ in parentheses, whereas adjusted $p$-values for each variable are on the right side of the plot. Filled black squares represent $\mathrm{HR}$ estimates, with relative $95 \% \mathrm{Cl}$ shown as horizontal lines with brackets.

Figure 5: SLFN11 is expressed in a subset of immune-related cells in tonsil and HGSOC tissues. Panel A: Representative images of CD3, CD8, SLFN11, CD20 and CD68 IHC on serial sections of tonsil tissue. Shown is a lymphoid follicle with the round- to oval shaped germinal center, the surrounding mantle zone and, at the outer layer of the lymphoid follicle, the paracortical zone. SLFN11 is mainly expressed in the germinal center, which is mostly composed of B-cells (CD20+) and macrophages/monocytes (CD68+), as well as in the T-cell rich (CD3+/CD8+) paracortical zone. Panels $\boldsymbol{B}$ and $\boldsymbol{C}$ : Representative images of CD3, CD8, SLFN11, CD20 and CD68 IHC on serial sections of tumor SLFN11 high- (Panel B) and low (Panel C) and stroma SLFN11 high cancers. The insets show nuclear SLFN11 in cytosolic/membrane-based CD3, CD8, CD20 and CD68 positive cells (indicated by arrows in Panel B and C). Scale bars, $100 \mu \mathrm{m}$ (Panel A) and $50 \mu \mathrm{m}$ (Panel B). The insets show a $3 x$ magnification of the representative images.

Figure 6: SLFN11 expression is associated with immune signatures in HGSOC. Left: Dendrogram representing the similarity between different immunologic signatures calculated in the TCGA ovarian cancer dataset ( $\mathrm{N}=302$ cases). $x$ axis represents the Ward's D2 distance. Signature names have superscript numbers to denote the publication they are derived from: 1: Senbabaoglu $Y$ et al., 
2016(Senbabaoglu et al., 2016); 2: Rody A et al., 2009(Rody et al., 2009); 3: Rody A et al., 2011(Rody et al., 2011); Garg AD et al., 2016(Garg et al., 2016); Teschendorff AE et al., 2010(Teschendorff et al., 2010); if not specified, signatures are from 6: Thorsson V et al., 2018(Thorsson et al., 2018). Right: lollipop plot of correlations between SLFN11 and the aforementioned signatures. $x$ axis represents the Spearman's correlation coefficient between SLFN11 expression in the TCGA ovarian cancer dataset and the investigated signatures, whereas individual dots are coloured by $-\log 10$ of the FDR of the correlation.

Figure 7: Proposed model for the SLFN11-cancer immunity cycle. Clockwise from bottom left: SLFN11 can be upregulated in cancer cells and tumor infiltrating immune cells by interferon signaling and possibly by other factors, such as SLFN11 promoter demethylation. SLFN11 high cancer cells are more prone to undergo immunogenic cell death spontaneously or following platinum or other DNA damaging agent's treatment(Garg et al., 2017). This, in turn, recruits T cells, which activate the interferon signaling cascade and consequently stimulate the upregulation of the MHC I and MHC II complexes, and induction of the cancer-immunity cycle. Increased SLFN11 in cancer cells may directly contribute to immune system activation(Murai et al., 2020) and interferon signaling may directly and indirectly induce cytoxicity in cancer cells(Mezzadra et al., 2019). Dashed arrows indicate relations that were not assessed in the present manuscript but derived from other publications. 
Table 1: Cohort demographics

\begin{tabular}{|l|l|l|l|}
\hline & PR & PS & p-value \\
\hline $\begin{array}{l}\text { Stage (n, percent) } \\
\text { IIIc } \\
\text { IVa }\end{array}$ & $11(85)$ & $13(87)$ & 1.000 \\
\hline Cycles (number, IQR) & $2(15)$ & $2(13)$ & \\
\hline & $7(6-8)$ & $7(6-9)$ & 1.000 \\
\hline PFI (months, 95\% Cl) & $4(2-6)$ & $11(9-$ n.r.) & n.c. \\
\hline $\begin{array}{l}\text { Age at diagnosis (years, } \\
\text { IQR) }\end{array}$ & $65.7(64.3-69.8)$ & $58.2(54.7-61.5)$ & 0.0026 \\
\hline
\end{tabular}

$\mathrm{PR}=$ platinum-resistant, $\mathrm{PS}=$ Platinum-sensitive, $\mathrm{IQR}=$ interquartile range, $\mathrm{PFI}=$ progression-free interval, n.r. $=$ not reached, n.c. $=$ not calculated . 


\section{Supplementary figure legends:}

Figure S1: Frequency distribution of SLFN11 H-scores in the analyzed HGSOC case set. Nuclear SLFN11 protein in cancer and non-cancer cells was blindly assessed with automated image analysis by Halo and quantified as an $\mathrm{H}$-score in all nuclear cells (panel A), cancer cells (panel B), and non-cancer cells (panel C). $y$ axis: frequency of samples within each $\mathrm{H}$-score bin; $x$ axis: H-score values, subdivided into increasing 30-unit bins.

Figure S2: SLFN11 is independently prognostic in HGSOC also when assessed in cancer or non-cancer cells only. Panels $\boldsymbol{A}$ and $\boldsymbol{C}$ : Waterfall plot showing SLFN11 protein levels in cancer (panel A) and non-cancer (panel C) cells, colored by platinum sensitivity: SLFN11 protein is reported as $\mathrm{H}$-score ( $y$ axis), whereas cases are reported by increasing values ( $x$ axis) and colored in red if platinum-refractory (PR) or light blue if non-refractory (NR). Panels $\boldsymbol{B}$ and $\boldsymbol{D}$ : Kaplan-Meier plots showing progression-free interval (PFI) stratified by SLFN11 cancer protein levels ("high" if $\mathrm{H}$-score > 5, "low" if < 5, panel B) and non-cancer protein levels ("high" if $\mathrm{H}$ score > 110, "low" if < 110, panel B). The progressed fraction of patients ( $y$ axis) is plotted against time expressed in months from the end of first-line chemotherapy, censored at 24 months ( $x$ axis). Numbers at risk are reported below the plots. Pvalues in the bottom left of the plots are from the Wald statistics for the univariable Cox's regression. Panels $\boldsymbol{E}$ and F: Forest plots of hazard ratios (x axis, in log scale) for SLFN11 protein in cancer (panel E) and non-cancer (panel F) cells together with variables retained by the multivariable model generated for overall SLFN11. Point HR estimates are reported below each variable together with $95 \%$ confidence intervals $(95 \% \mathrm{Cl})$ in parentheses, whereas adjusted $\mathrm{p}$-values for each variable are on the right side of the plot. Filled black squares represent HR estimates, with relative $95 \% \mathrm{Cl}$ shown as horizontal lines with brackets.

Figure S3: SLFN11 is expressed in a subset of leukocyte subpopulations. Panel A: Scatterplot representing SLFN11 expression (log-transformed normalized counts, $y$ axis) as a function of GSEA macrophage enrichment score (ES, $x$ axis) in TCGA OVCAR dataset $(\mathrm{N}=302)$; $\rho$ is the Spearman's correlation coefficient, the 
least squares regression are represented by the red lines, whereas dots are measurements of SLFN11 expression by ES in individual samples. Panel B: Box plots of publicly available RNA-sequencing results (GEO accession number GSE60424) for SLFN11 in sorted leukocyte subpopulations from patients. NK: natural killer cells. GEO: gene expression omnibus.

\section{Figure S4: Cancer cellularity is negatively correlated with SLFN11 in ovarian} cancer. Panel A: Scatterplot representing SLFN11 transcript (log-transformed normalized counts, $y$ axis) as a function of cancer cellularity inferred using ESTIMATE ( $x$ axis) in TCGA OVCAR dataset $(\mathrm{N}=302)$. Panel B: Scatterplot representing SLFN11 protein measured in all nuclear cells (overall H-score, $y$ axis) as a function of cancer cellularity measured by HALO ( $x$ axis) in our cohort $(\mathrm{N}=27)$. $\rho$ is the Spearman's correlation coefficient, the least squares regression are represented by the red lines, whereas dots are measurements of SLFN11 protein levels by cancer cellularity in individual samples.

Figure S5: SLFN11 in tonsil tissues. Representative images of IGG IHC (negative control) and serial sections of tonsil tissue. Shown is a lymphoid follicle with the ovalshaped germinal center, the surrounding mantle zone and, at the outer layer of the lymphoid follicle, the paracortical zone. The insets show H\&E stained tonsil tissue and confirm the absence of signal in the negative control sample. 
bioRxiv preprint doi: https //doi org/10.1101/2020 05.22.110593; this version posted May 26, 2020. The copyright holder for this preprint (which was not certified by peer review) is the author/funder, who has granted bioRxiv a license to display the preprint in perpetuity. It is made available under aCC-BY-NC-ND 4.0 International license.

Fig. 1

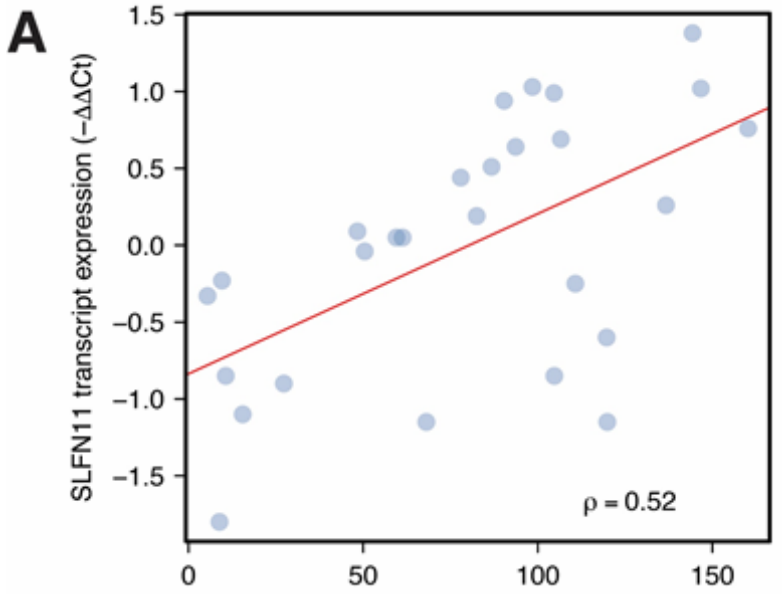

SLFN11 protein levels (overall H-score)

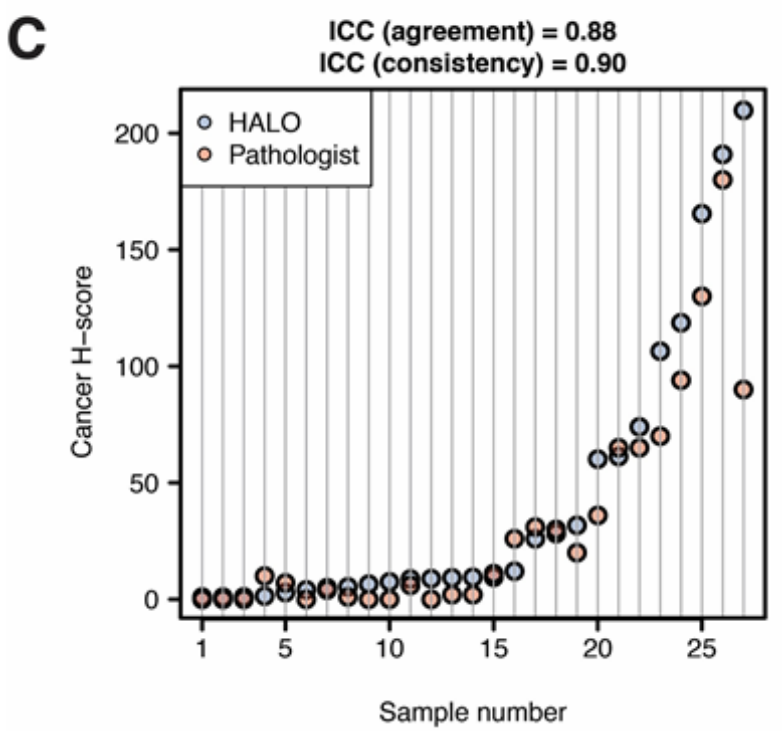

B

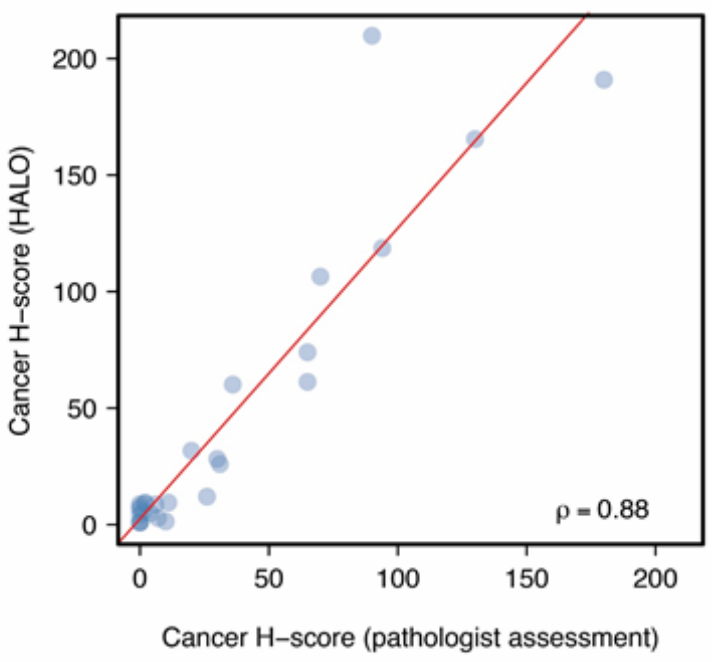

D

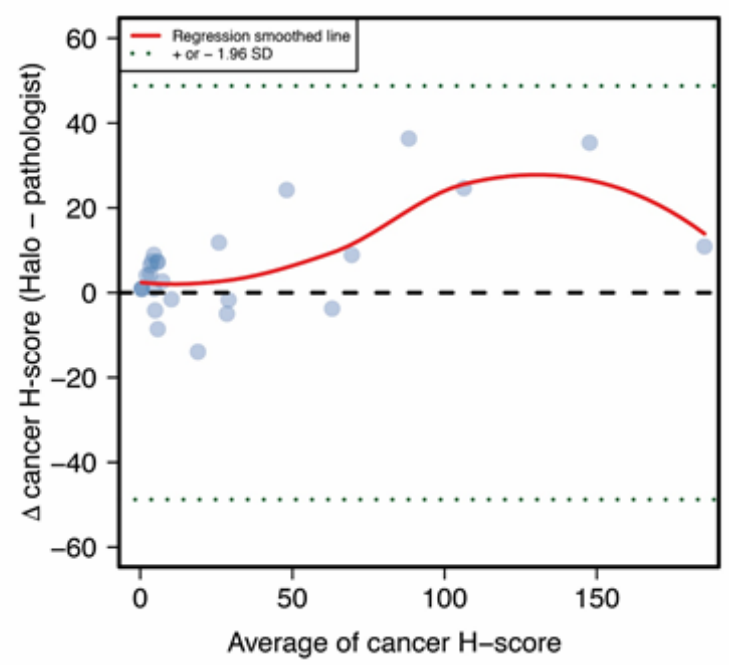


bioRxiv preprint doi: https://doi.org/10.1101/2020.05.22.110593; this version posted May 26, 2020. The copyright holder for this preprint (which was not certified by peer review) is the author/funder, who has granted bioRxiv a license to display the preprint in perpetuity. It is made available under aCC-BY-NC-ND 4.0 International license.

\section{Fig. 2}

A

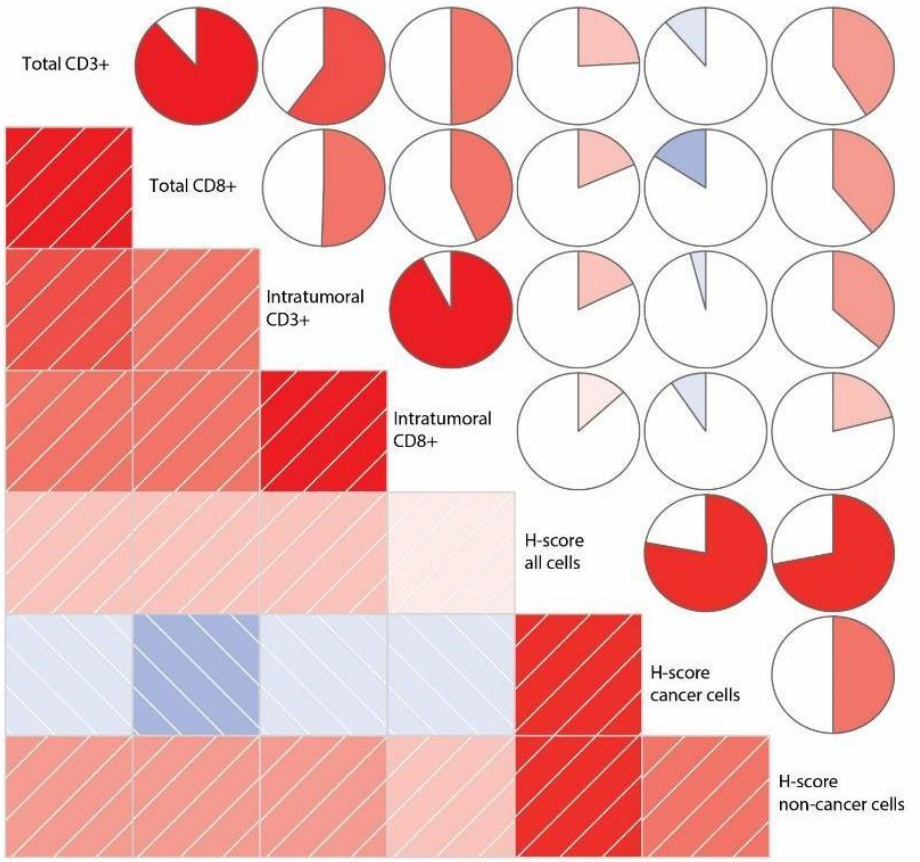

B

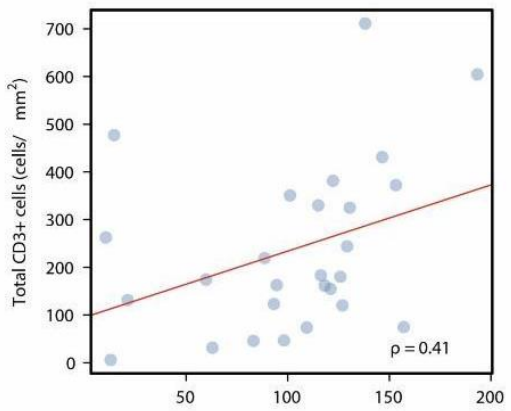

C

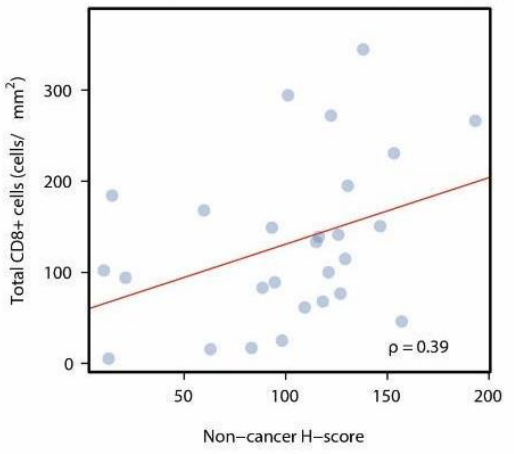

D
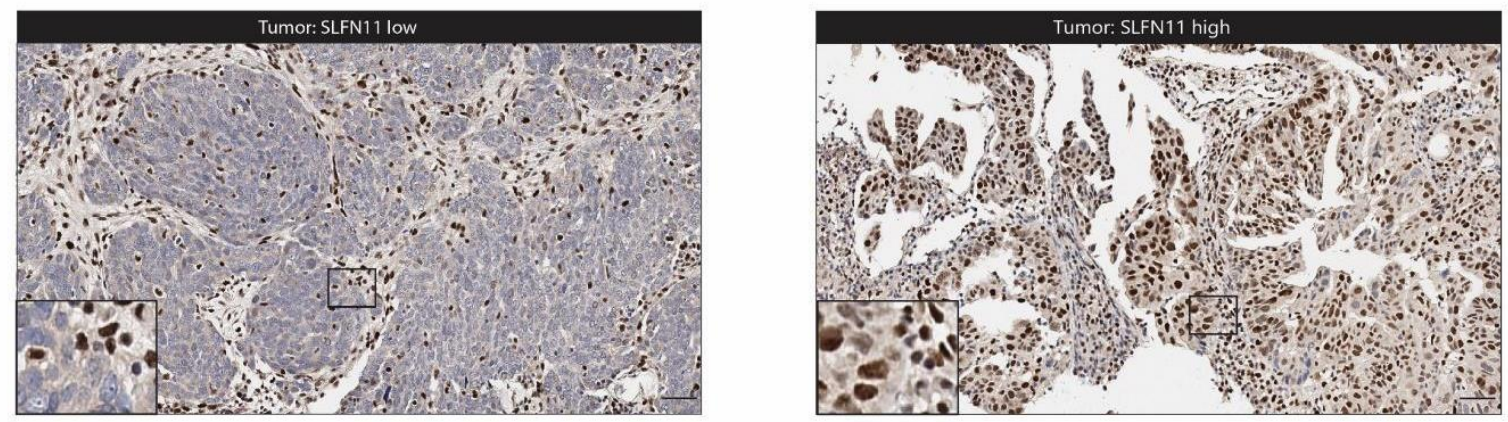
bioRxiv preprint doi: https://doi.org/10.1101/2020.05.22.110593; this version posted May 26, 2020. The copyright holder for this preprint (which was not certified by peer review) is the author/funder, who has granted bioRxiv a license to display the preprint in perpetuity. It is made available under aCC-BY-NC-ND 4.0 International license.

Fig. 3

A

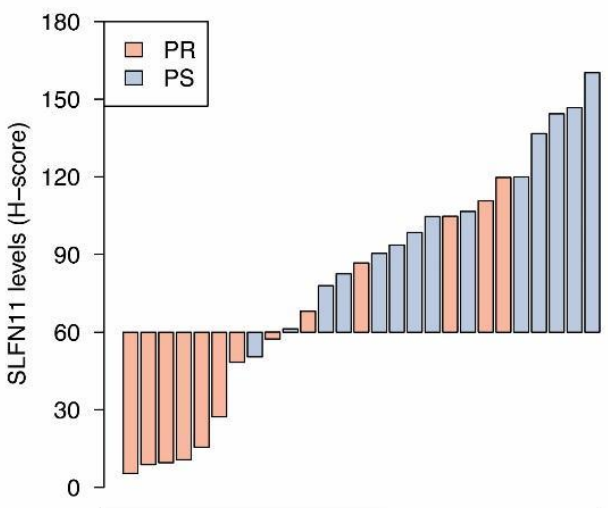

B

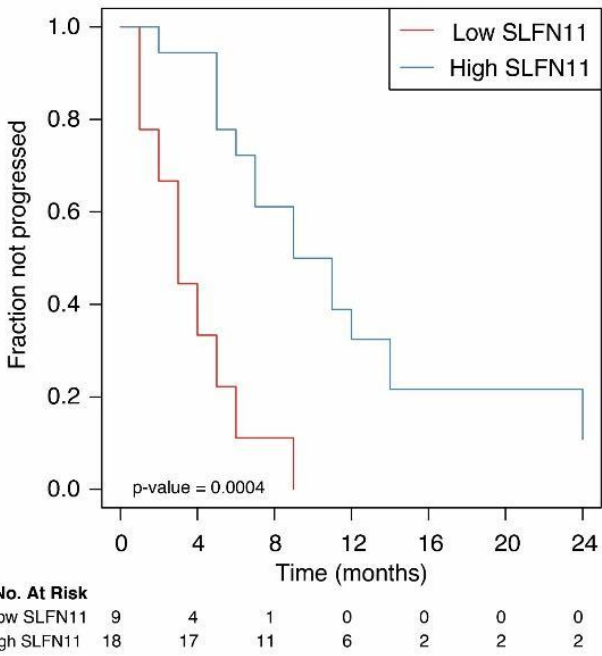

High SLFN11 18
C

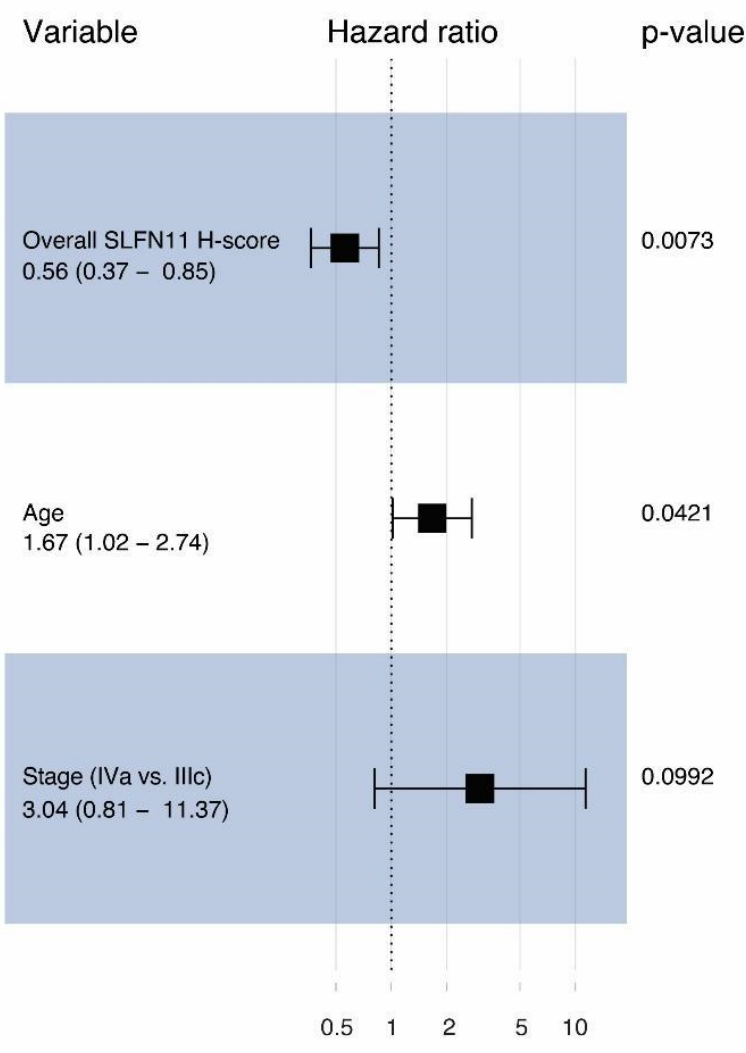


bioRxiv preprint doi: https://doi.org/10.1101/2020.05.22.110593; this version posted May 26, 2020. The copyright holder for this preprint (which was not certified by peer review) is the author/funder, who has granted bioRxiv a license to display the preprint in perpetuity. It is made available under aCC-BY-NC-ND 4.0 International license.

Fig. 4

A

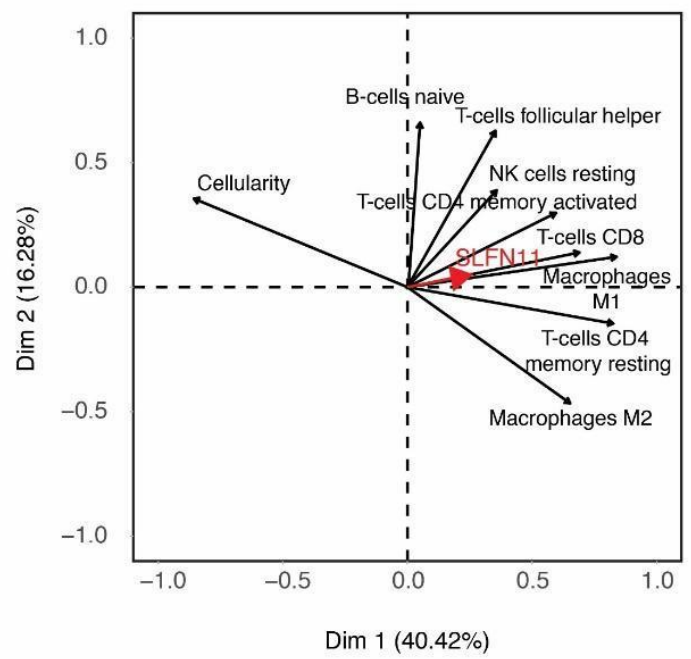

B

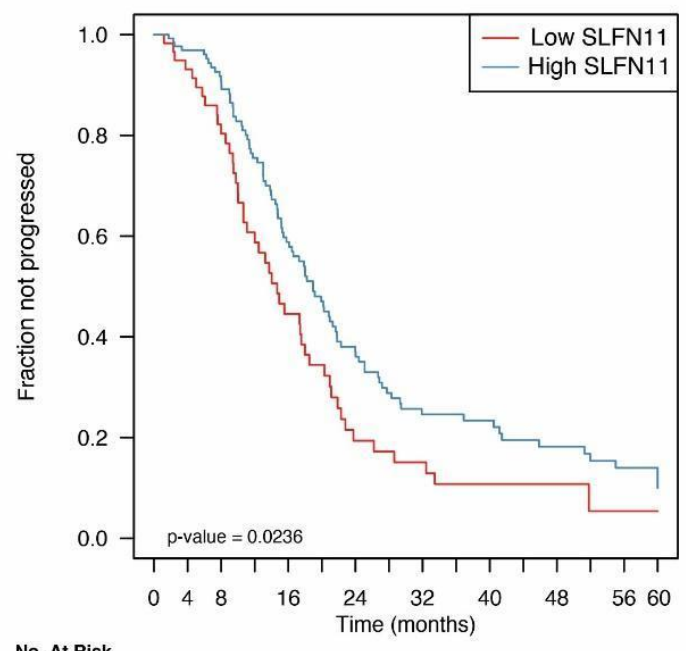

C

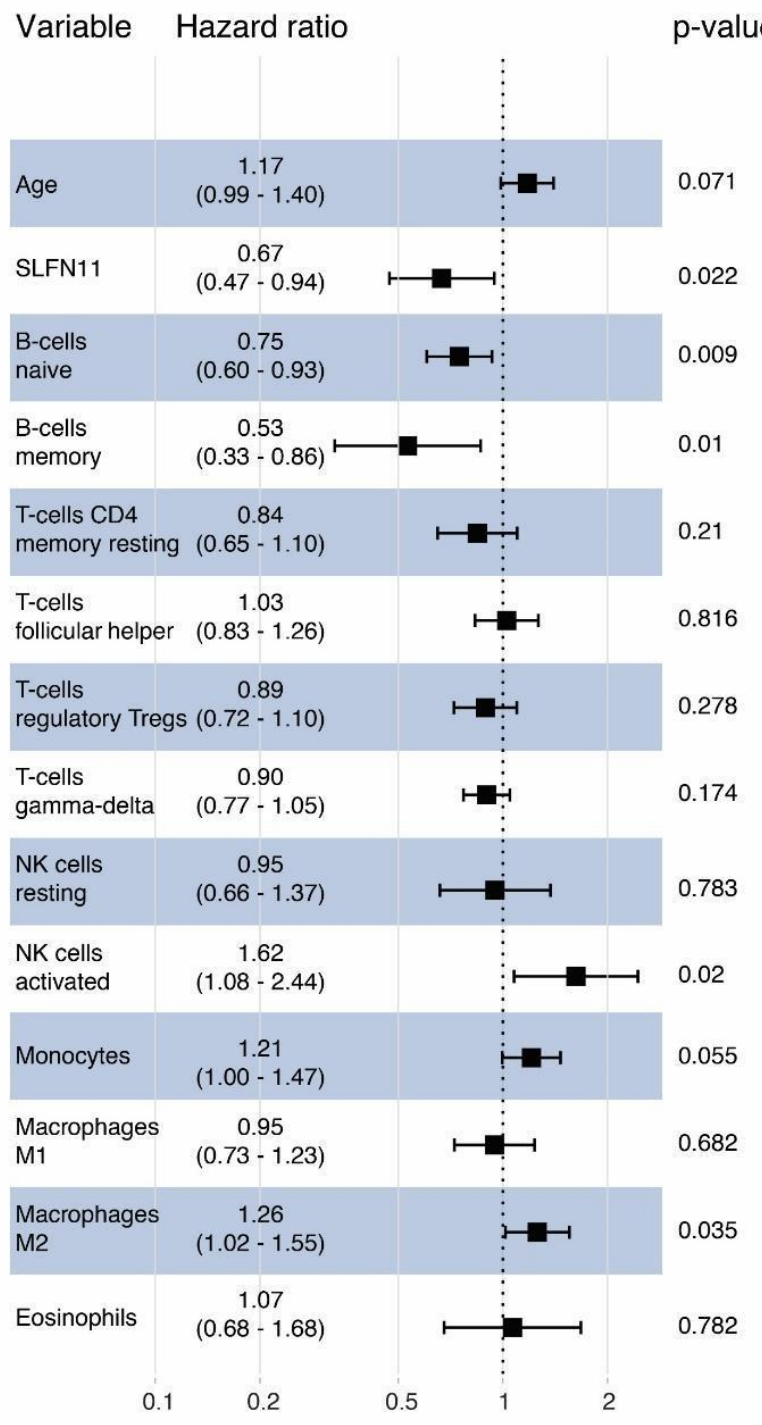

Low SLFN11 $55 \begin{array}{lllllllllllllll}52 & 42 & 29 & 22 & 16 & 9 & 8 & 7 & 4 & 4 & 4 & 3 & 1 & 1 & 1\end{array}$

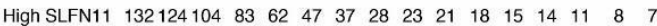


bioRxiv preprint doi: https://doi.org/10.1101/2020.05.22 110593. this version posted May 26, 2020. The copyright holder for this preprint (which was not certified by peer review) is the author/funder, who has granted bioRxiv a license to display the preprint in perpetuity. It is made available under aCC-BY-NC-ND 4.0 International license.

Fig. 5

A
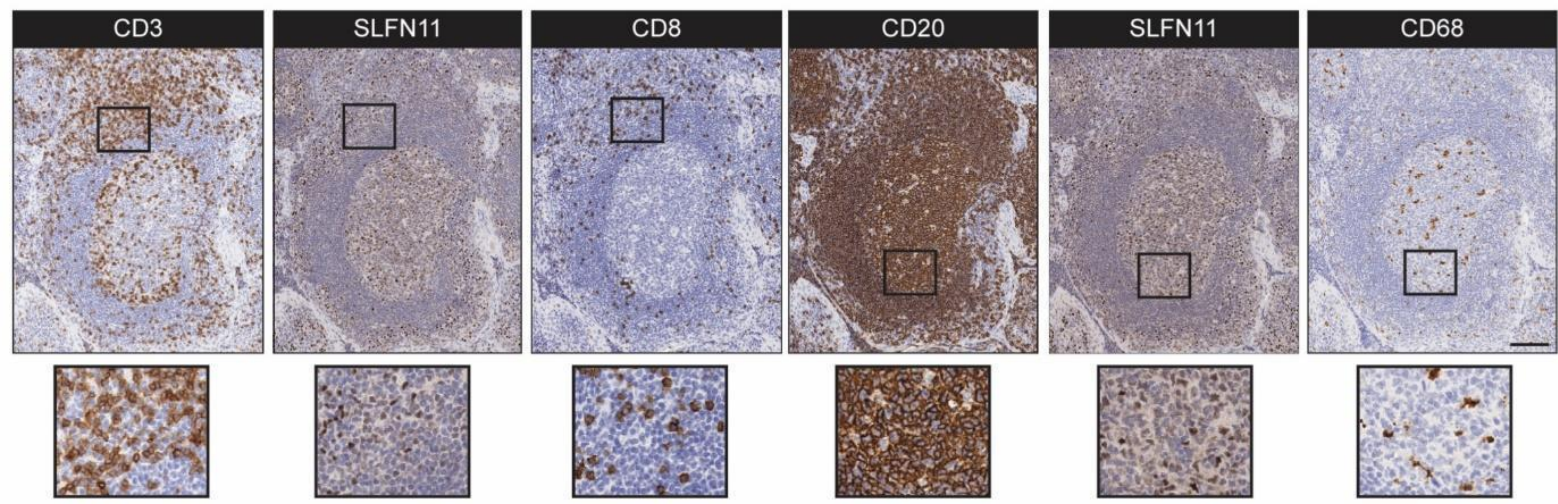

B
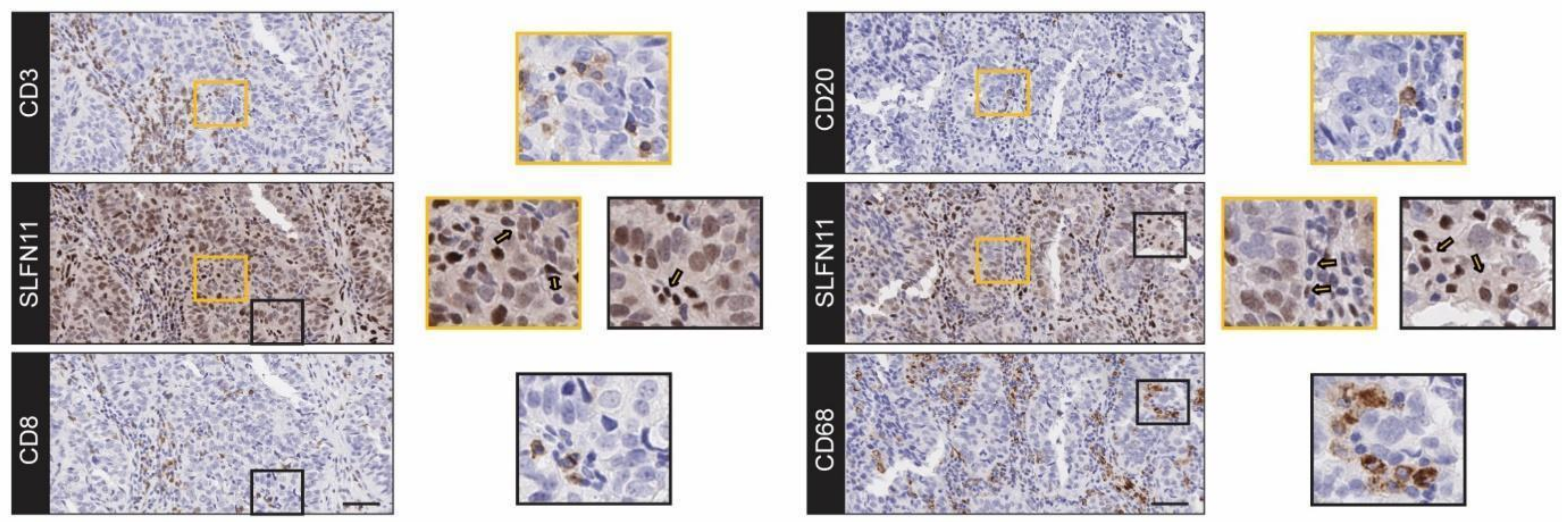

C
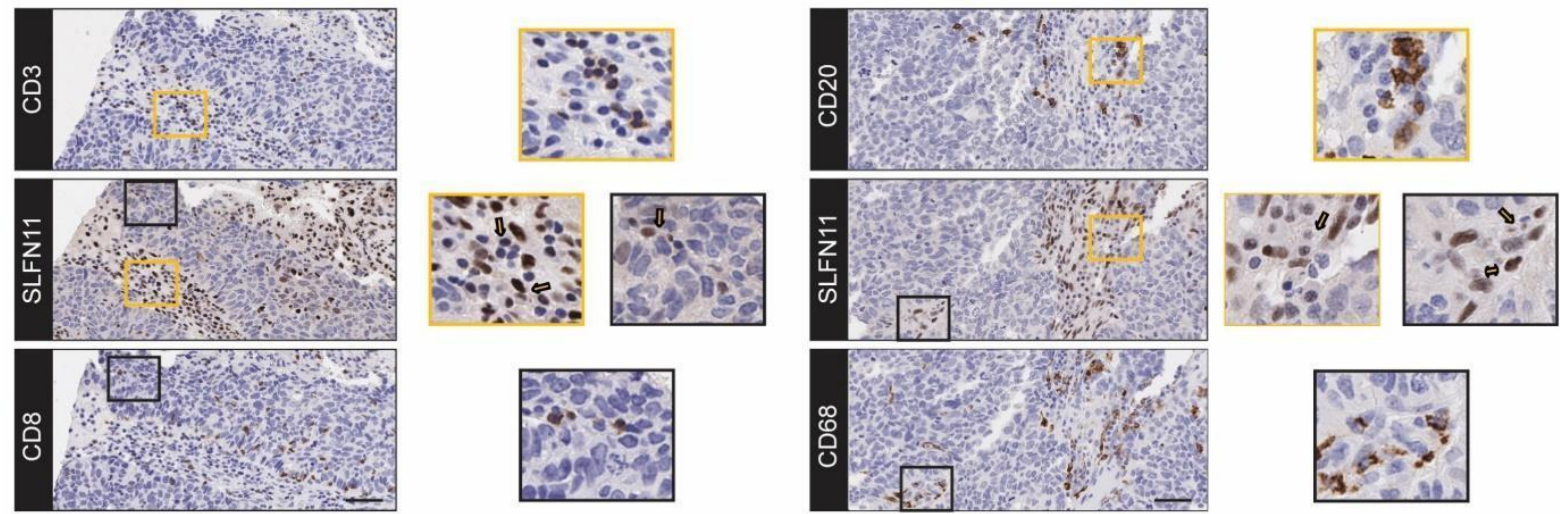
bioRxiv preprint doi: https://doi. org/10.1101/2020.05.22.110593; this version posted May 26, 2020. The copyright holder for this preprint (which was not certified by peer review) is the author/funder, who has granted bioRxiv a license to display the preprint in perpetuity. It is made available under aCC-BY-NC-ND 4.0 International license.

Fig. 6

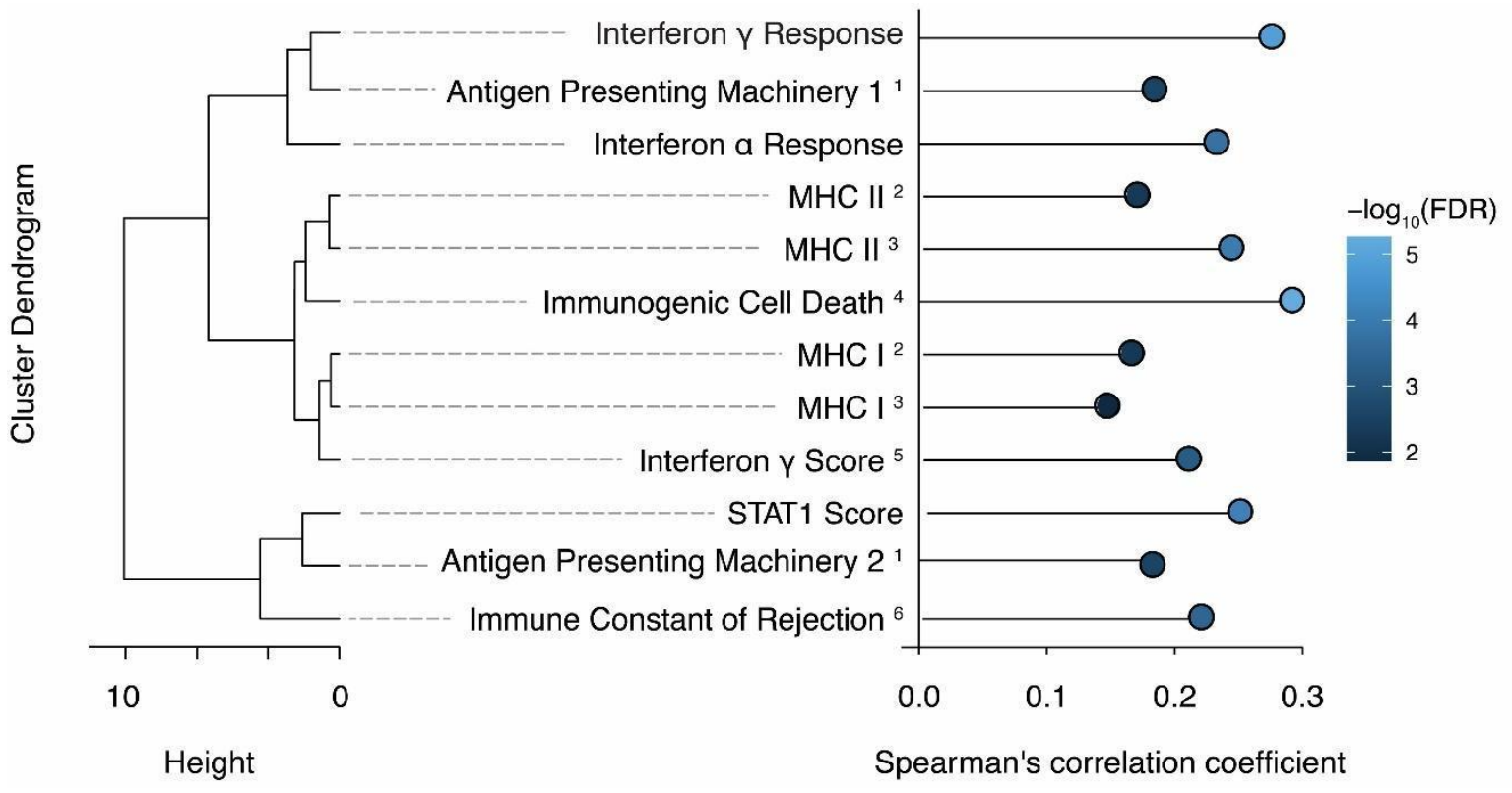


bioRxiv preprint doi: https://doi.org/10.1101/2020.05.22.110593; this version posted May 26, 2020. The copyright holder for this preprint (which was not certified by peer review) is the author/funder, who has granted bioRxiv a license to display the preprint in perpetuity. It is made available under aCC-BY-NC-ND 4.0 International license.

Fig. 7

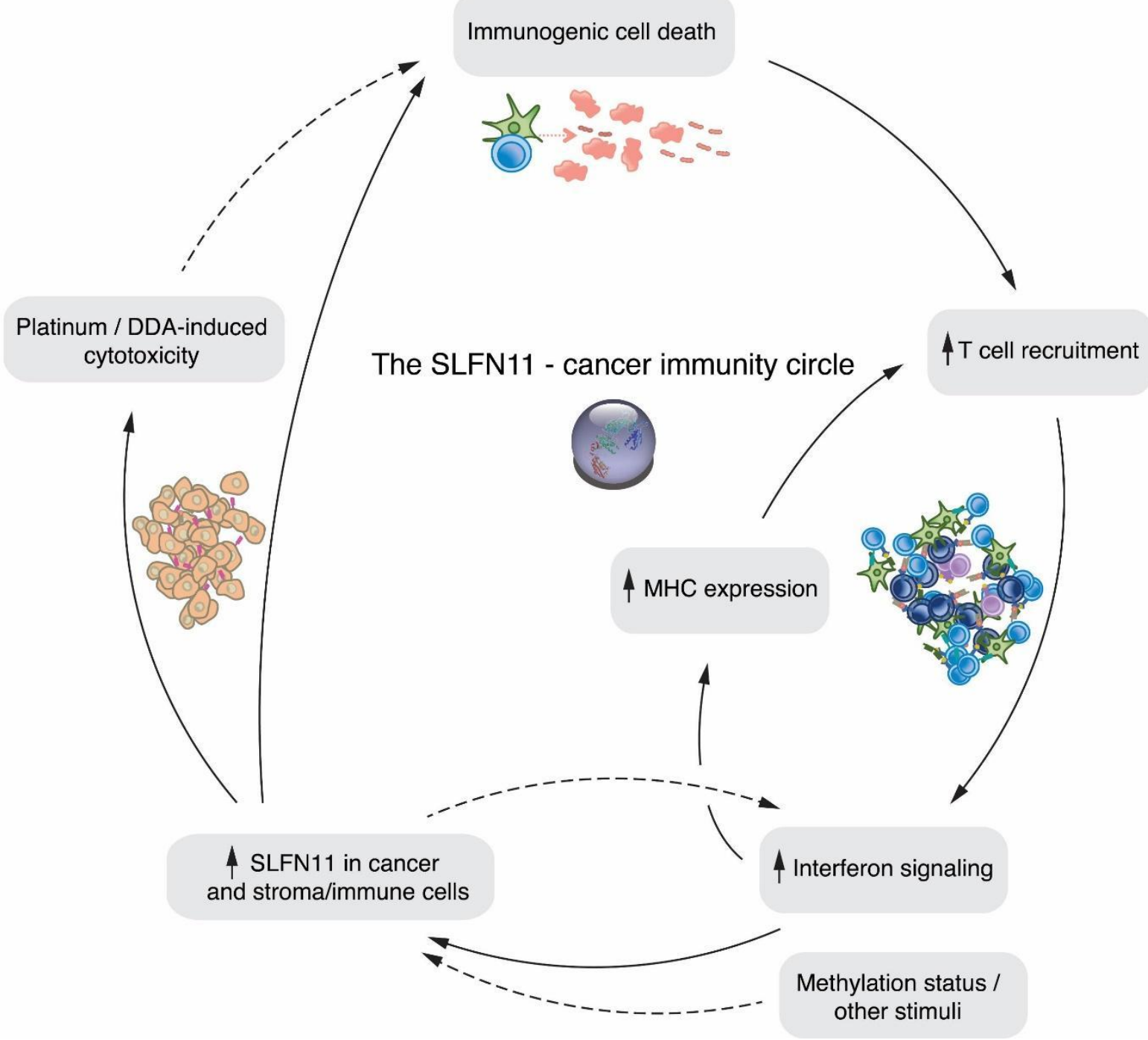


bioRxiv preprint doi: https://doi.org/10.1101/2020.05.22.110593; this version posted May 26, 2020. The copyright holder for this preprint (which was not certified by peer review) is the author/funder, who has granted bioRxiv a license to display the preprint in perpetuity. It is made available under aCC-BY-NC-ND 4.0 International license.

Fig. $\$ 1$

A

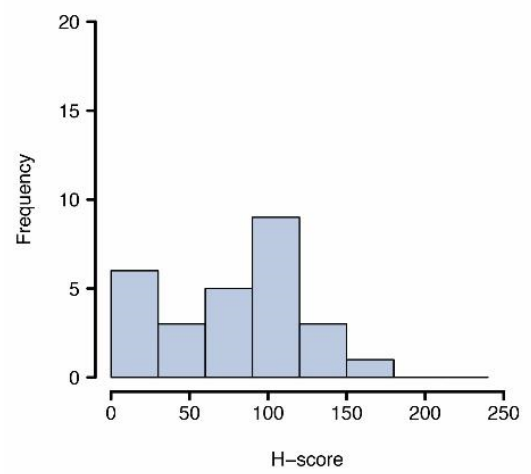

B

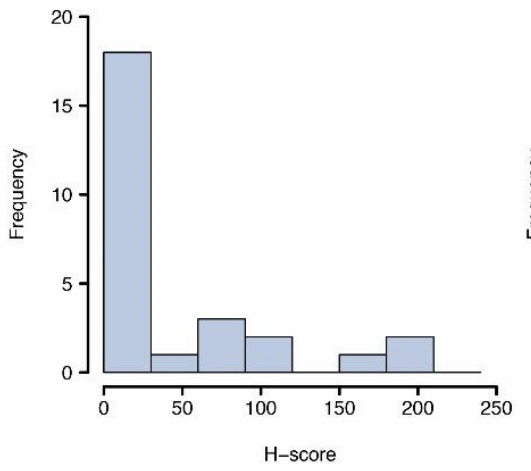

C

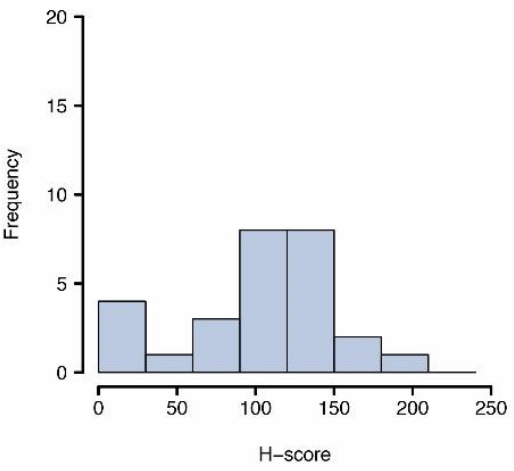


bioRxiv preprint doi: https://doi.org/10.1101/2020 05.22.110593. this version posted May 26, 2020. The copyright holder for this preprint

Fig. S2
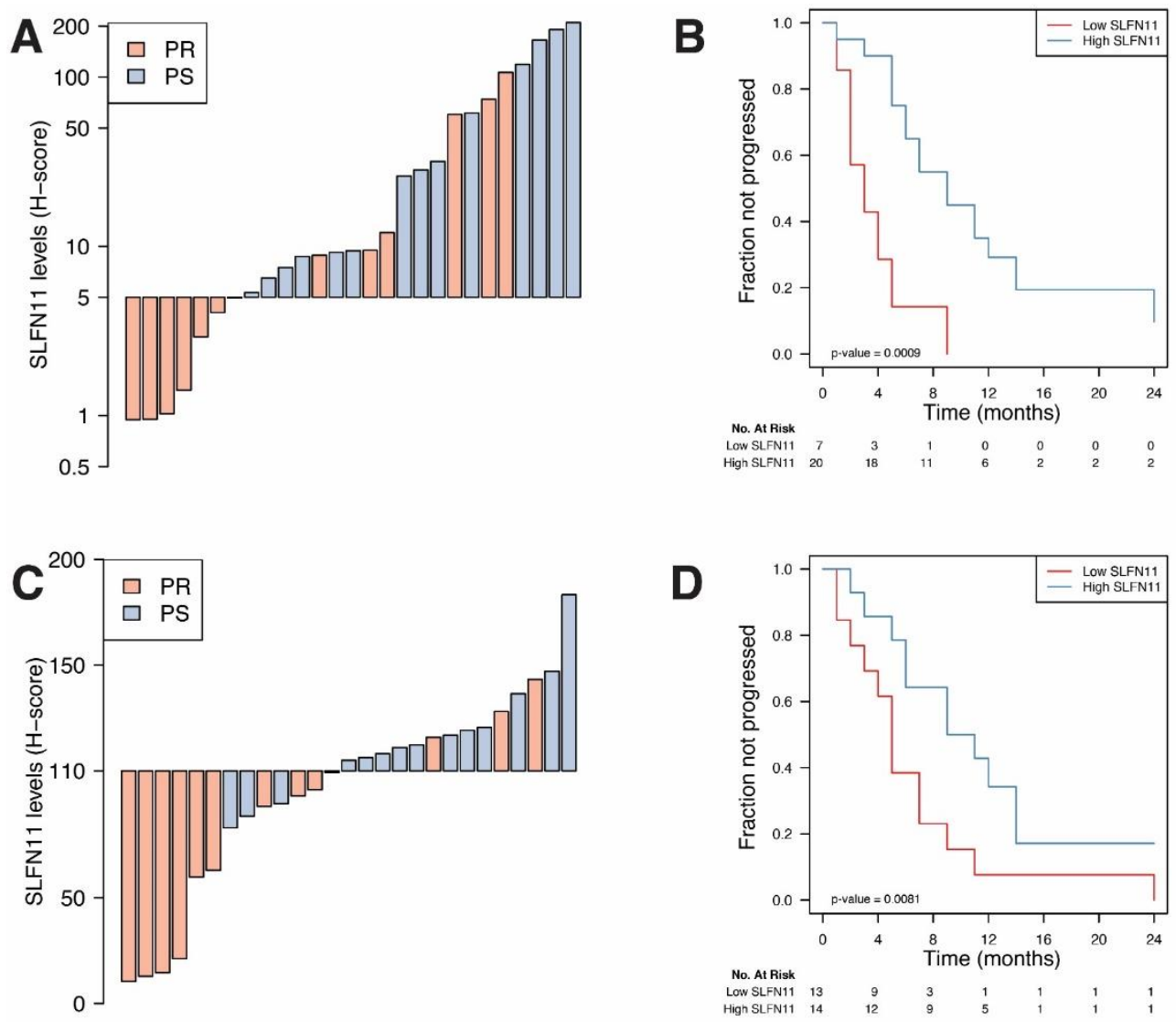

$\mathbf{E}$

$\mathbf{F}$

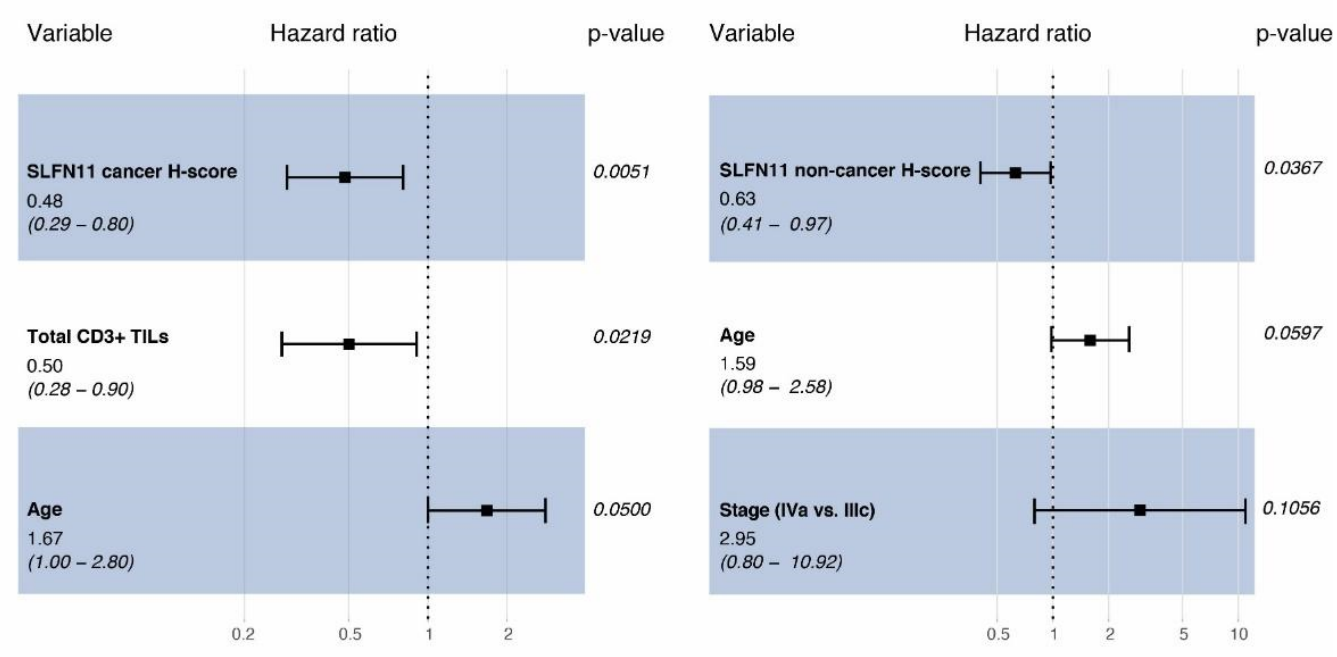


bioRxiv preprint doi: https://doi.org/10.1101/2020 05.22 110593; this version posted May 26, 2020. The copyright holder for this preprin (which was not certified by peer review) is the author/funder, who has granted bioRxiv a license to display the preprint in perpetuity. It is made available under aCC-BY-NC-ND 4.0 International license.

Fig. S3

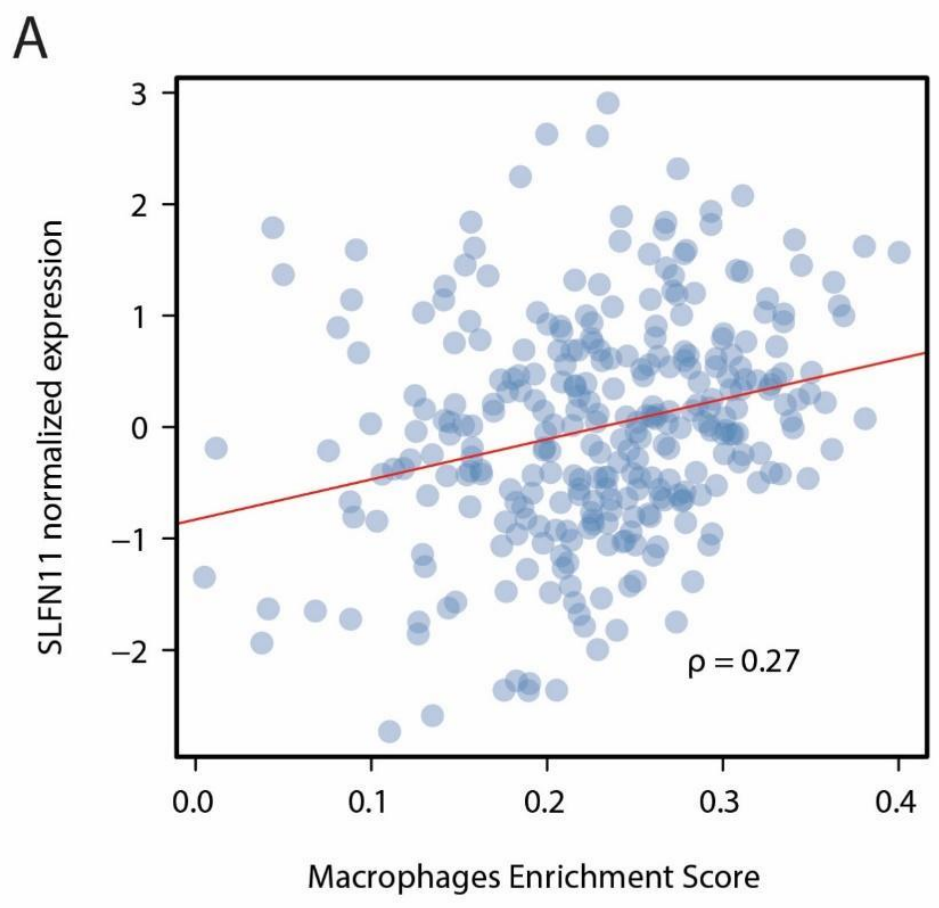

B

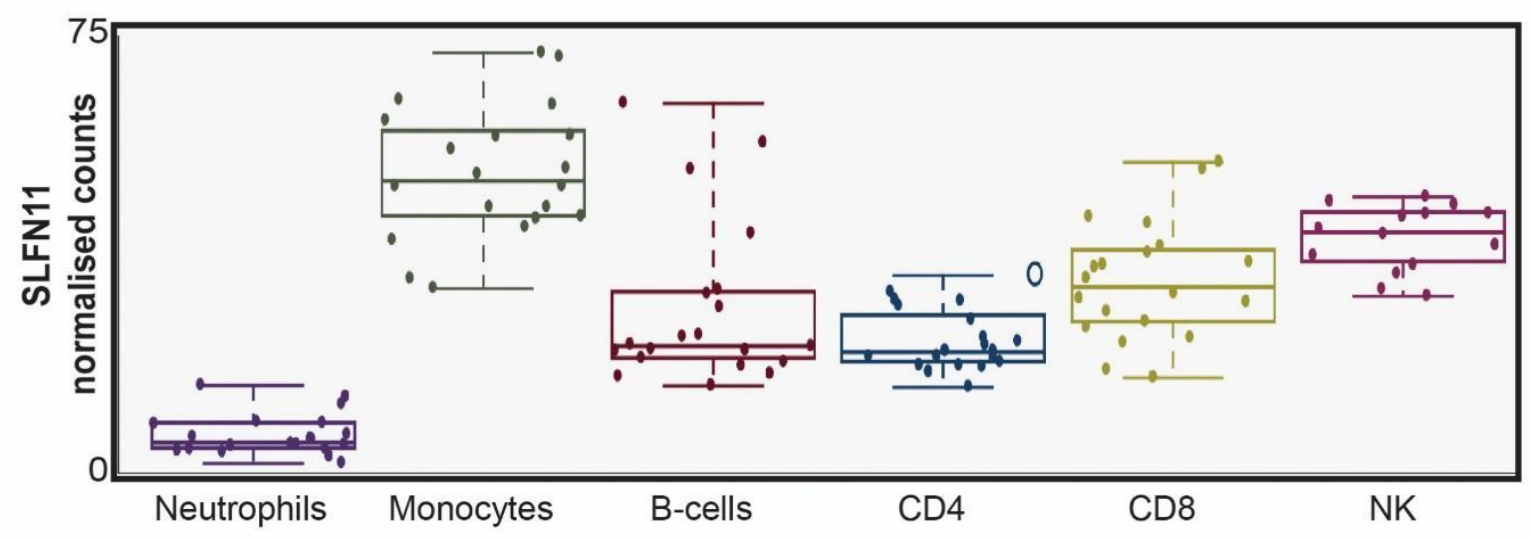


bioRxiv preprint doi: https://doi.org/10.1101/2020.05.22.110593; this version posted May 26, 2020. The copyright holder for this preprint (which was not certified by peer review) is the author/funder, who has granted bioRxiv a license to display the preprint in perpetuity. It is made available under aCC-BY-NC-ND 4.0 International license.

Fig. 54

A

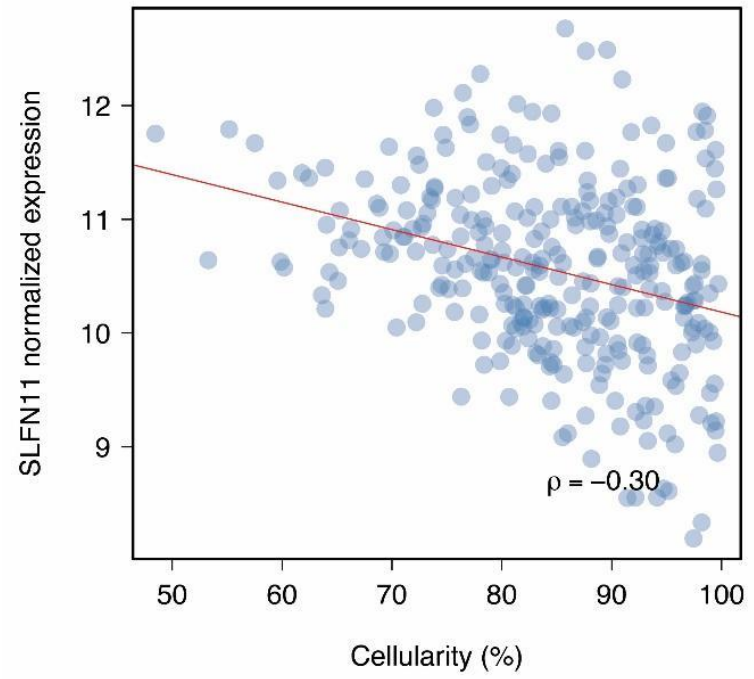

B

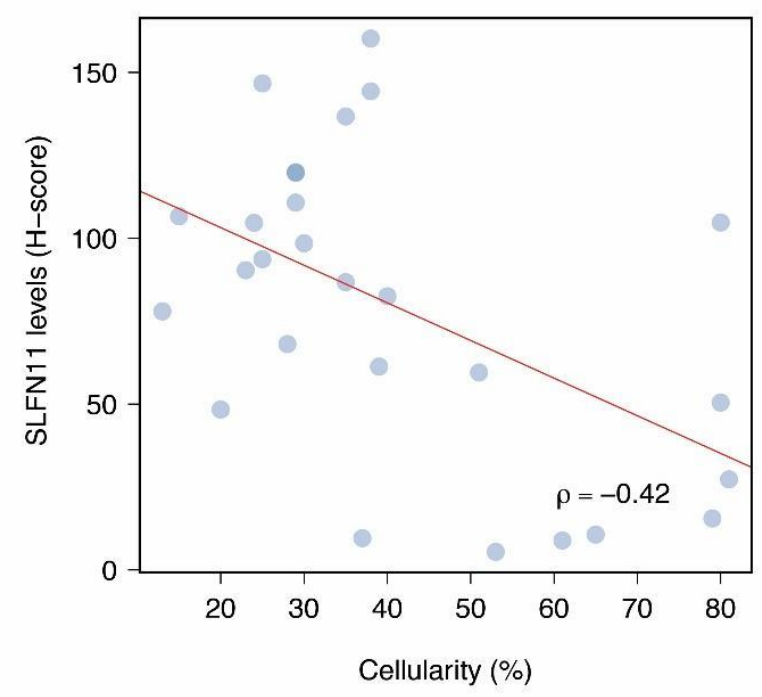


bioRxiv preprint doi: https://doi.org/10.1101/2020.05.22.110593; this version posted May 26, 2020. The copyright holder for this preprint (which was not certified by peer review) is the author/funder, who has granted bioRxiv a license to display the preprint in perpetuity. It is made available under aCC-BY-NC-ND 4.0 International license.

Fig. S5
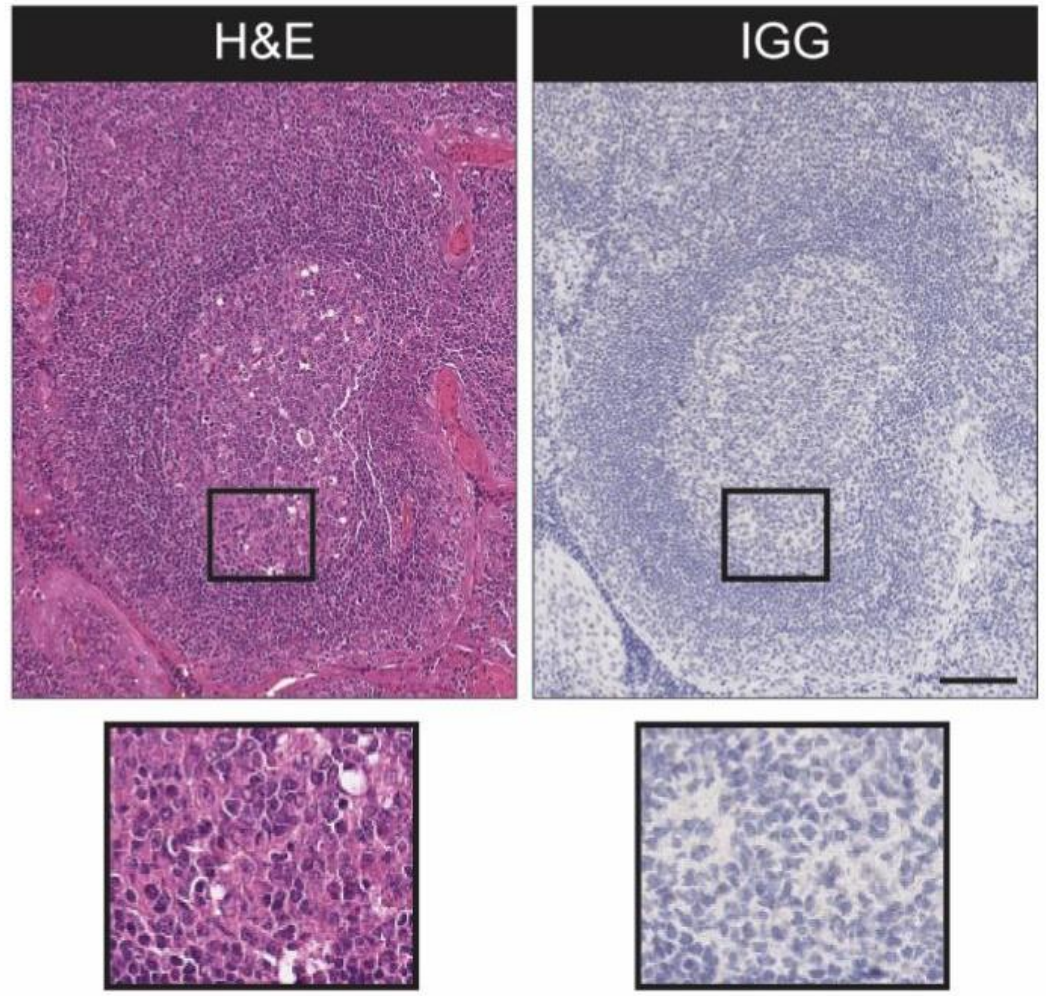